\title{
Peroxiredoxin-2 Upregulated by NF- $\kappa$ B Attenuates Oxidative Stress During the Differentiation of Muscle-Derived C2C12 Cells
}

\author{
Hyeran Won, Sangbin Lim, Miran Jang, Yeonghwan Kim, Md Abdur Rashid, K.R. Jyothi, \\ Amarjargal Dashdorj, Insug Kang, Joohun Ha, and Sung Soo Kim
}

\begin{abstract}
Aim: Many studies have reported that the generation of reactive oxygen species (ROS) increases during the differentiation of muscle-derived $\mathrm{C} 2 \mathrm{C} 12$ cells. Peroxiredoxin-2 (Prx-2) is an abundant mammalian enzyme that protects against oxidative stress. However, the role of Prx-2 in muscle differentiation has not been investigated. Results: In this study, we demonstrated that Prx-2 expression increases during muscle differentiation and regeneration in response to exogenous $\mathrm{H}_{2} \mathrm{O}_{2}$. This increase occurs only in myoblast cell lines because no increase in Prx-2 expression was observed in the NIH3T3, MEF, Chang, or HEK293 cell lines. The antioxidants, N-acetyl L-cysteine (NAC) and 4,5-dihydroxy-1,3-benzenedisulfonic acid (Tiron), both suppressed myogenesis and Prx-2 expression. Moreover, Prx-2 was upregulated at the transcriptional level by NF- $\kappa$ B during the differentiation of muscle-derived C2C12 cells. We also found that inhibition of phosphatidylinositol 3-kinase (PI3K) blocks NF- $\kappa \mathrm{B}$ activation and suppresses Prx-2 expression. Interestingly, Prx-2 knockdown increased the expression levels of other antioxidant enzymes, including all of the other Prx family member, thioredoxin-1 (Trx-1) and catalase, but also enhanced the accumulation of endogenous ROS during muscle differentiation. Innovation: In this study, we demonstrated for the first time that Prx-2 is unregulated during the muscle differentiation and regeneration. Conclusion: Prx-2 is upregulated via the PI3K/NF- $\kappa$ B pathway and attenuates oxidative stress during muscle differentiation and regeneration. Antioxid. Redox Signal. 16, 245-261.
\end{abstract}

\section{Introduction}

$\mathbf{M}$ USCLE DIFFERENTIATION consists of a number of steps, including an irreversible withdrawal from the cell cycle, the induction of muscle-specific genes, such as myosin heavy chain (MHC), and the fusion of myocytes into multinucleated myotubes (7, 33). Intracellular signaling pathways control these complex processes and phosphatidylinositol 3-kinase (PI3K) is one of the crucial signaling molecules involved in muscle differentiation. PI3K affects downstream targets such as Akt, Rac, P70 ${ }^{\mathrm{S} 6 \mathrm{~K}}$, phospholipase C- $\gamma 1$ (PLC- $\gamma 1$ ), and mTOR (20), all of which are major signaling molecules that mediate myogenesis. Insulin and insulin-like growth factor (IGF) -1 and -2 are the best characterized ligands that stimulate muscle differentiation via the PI3K pathway $(7,20)$.

Peroxiredoxins (Prxs) are a novel group of peroxidases with high antioxidant activity, and some members of this family can affect cell proliferation, immune responses (31),

\section{Innovation}

It has not been investigated if Prx-2 is unregulated at the transcriptional level in response to oxidative stress. In this study, we first showed that Prx-2 is upregulated during muscle differentiation and regeneration. We further demonstrated that Prx-2 is upregulated by the PI3K/NF- $\kappa \mathrm{B}$ pathway during muscle differentiation. Interestingly, the overexpression of Prx-2 prevents both myogenesis and ROS production. In contrast, the inhibition of Prx-2 expression induces the compensatory expressions of other antioxidant enzymes, and enhances muscle differentiation and ROS production. In conclusion, our findings provide a critical insight into the transcriptional regulatory mechanisms of Prx-2 via PI3K/NF- $\kappa$ B during muscle differentiation and regeneration.

Department of Biochemistry and Molecular Biology (BK21 project), Medical Research Center for Bioreaction to Reactive Oxygen Species and Biomedical Science Institute, School of Medicine, Kyung Hee University, Seoul, Korea. 
A

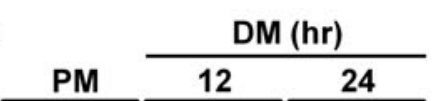

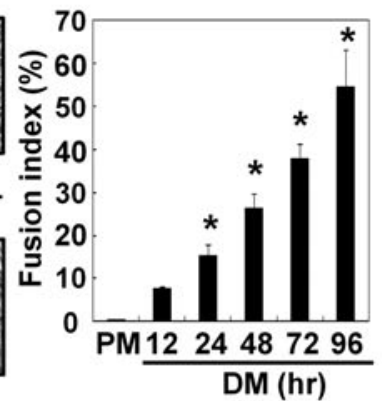

B
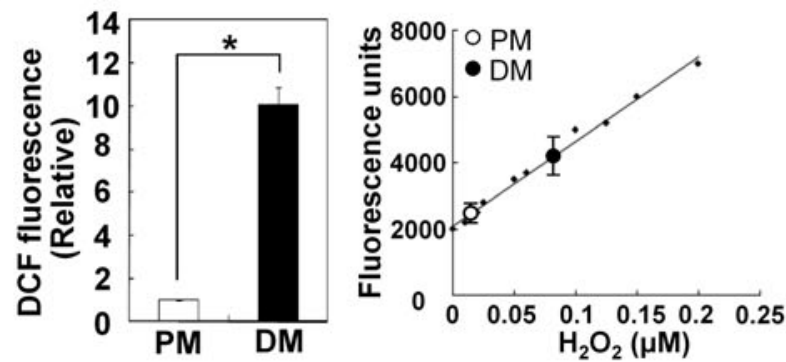

C

(-) $\beta$-mer.

(+) $\beta$-mer.

PM $\frac{\mathrm{DM}(\mathrm{hr})}{1224487296}$ PM $\frac{\mathrm{DM}(\mathrm{hr})}{1224487296}$

Myogenin - - - - - - - -
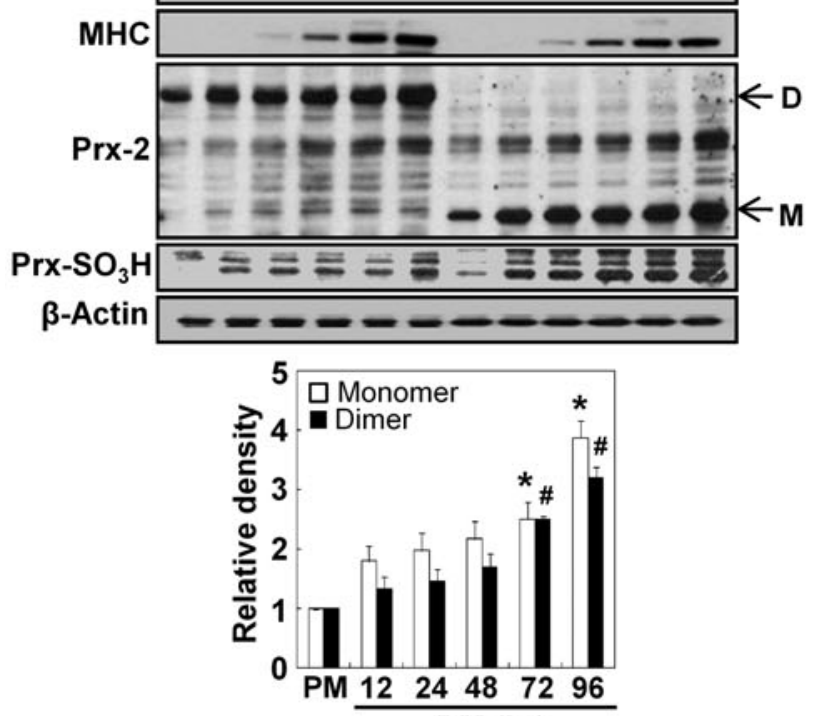

D

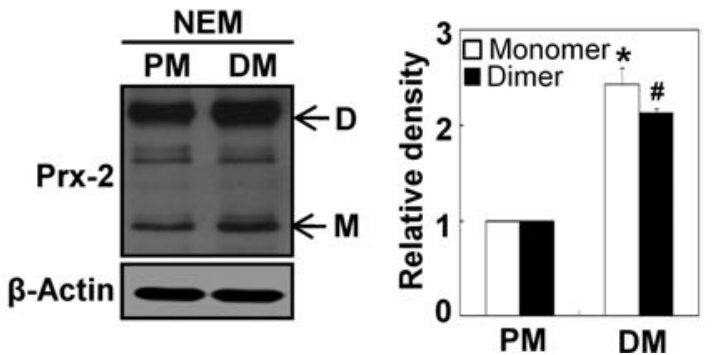

E

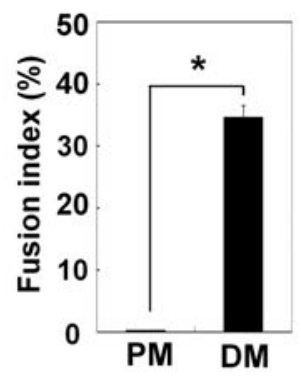

$\mathbf{F}$
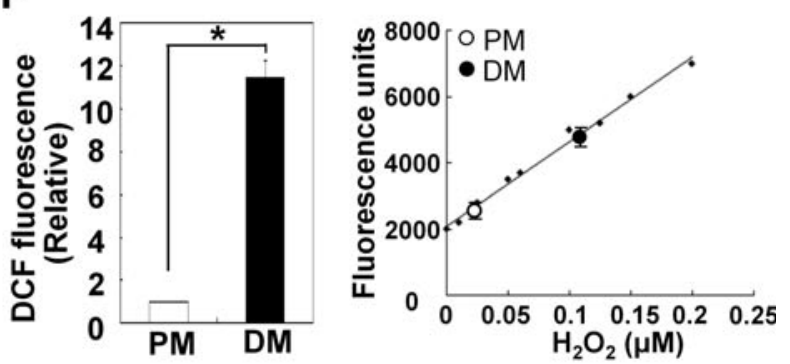

G

\section{(-) $\beta$-mer. (+) $\beta$-mer.}
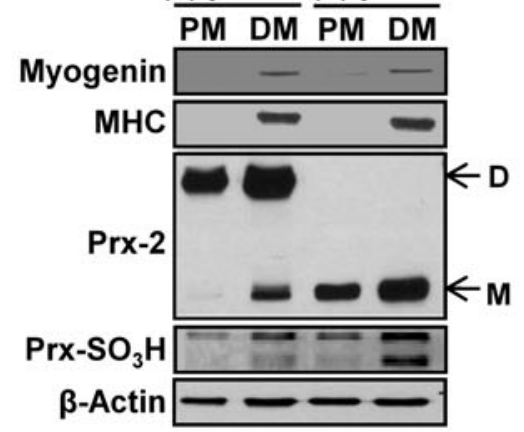

H
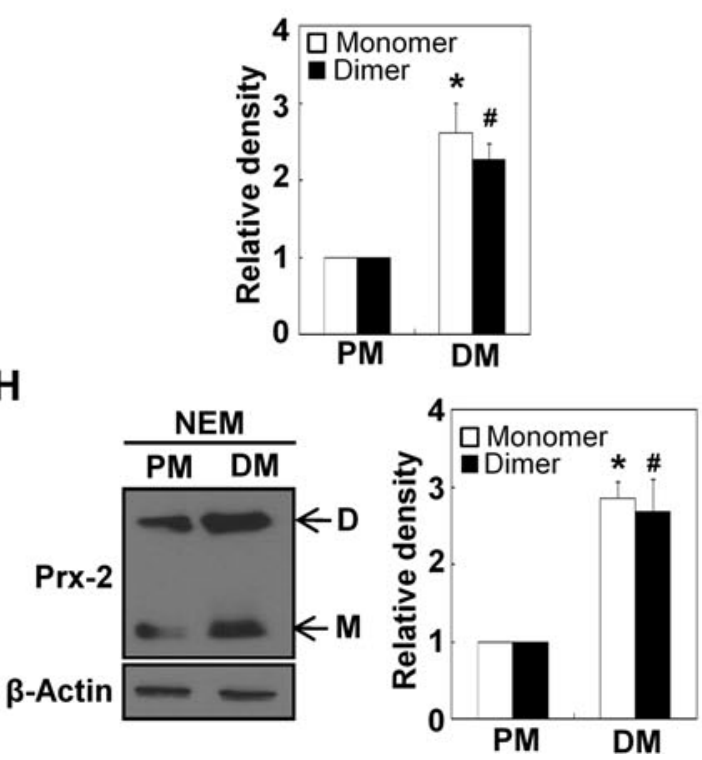
and apoptosis and differentiation (13). In mammals, six family members (Prx1-6) have been identified; these Prxs can be classified as typical 2-Cys, atypical 2-Cys, or 1-Cys Prxs based on the locations of their cysteine residues. Prx-2 belongs to the typical 2-Cys class and is a predominantly cytoplasmic Prx $(29,30)$; Prx-2 has redox-active cysteine residues that participate in the reduction of $\mathrm{H}_{2} \mathrm{O}_{2}$. Therefore, in the catalytic cycle, the conserved peroxidatic cysteine $\left(\mathrm{C}_{\mathrm{p}}\right)$ residue of Prx-2 is oxidized to cysteine sulfenic acid (Cys-SOH), which subsequently interacts with the resolving cysteine $\left(C_{r}\right)$ of another Prx-2 to form disulfide bonds between the two molecules, resulting in a homodimer that is subsequently reduced by thioredoxin (Trx). In general, Prx-2 is present in its reduced monomeric form together with Prx-2 disulfide catalytic cycle intermediates. In contrast, under oxidative stress, Prx-SOH is further oxidized by peroxide to sulfinic $\left(-\mathrm{SO}_{2} \mathrm{H}\right)$ and sulfonic acids $\left(-\mathrm{SO}_{3} \mathrm{H}\right)$, thereby causing the inactivation of its peroxidase activity. Prx- $\mathrm{SO}_{2} \mathrm{H}$ and $\mathrm{Prx}-\mathrm{SO}_{3} \mathrm{H}$, which are hyperoxidized Prx-2, can be slowly converted back to the reduced state by sulfiredoxin (Srx) (26).

$\mathrm{H}_{2} \mathrm{O}_{2}$ is an important regulator of signal messenger molecule for various pathways (34). We have previously demonstrated that $\mathrm{H}_{2} \mathrm{O}_{2}$ is an essential mediator of muscle differentiation (7). However, it is still not clear whether myogenesis is associated with the function of Prx-2 as an $\mathrm{H}_{2} \mathrm{O}_{2}$ scavenger; therefore we investigated the potential roles of Prx-2 in myogenesis.

In addition, the previous study showed that the $\mathrm{H}_{2} \mathrm{O}_{2}$ that is generated during muscle differentiation regulates nuclear factor kappa B (NF- $\kappa \mathrm{B})$ activation $(17,27,28)$. During redox events, NF- $\kappa \mathrm{B}$ is regulated by oxidative stress (12). NF- $\kappa \mathrm{B}$ consists of five subunits: p65, RelB, c-Rel, p105/p50, and p100/ $\mathrm{p} 52$, and these subunits are conserved and exist as hetero- or homodimers (8). The p65/p50 heterodimer is the most abundant NF- $\kappa \mathrm{B}$ complex in the cell and is present in the cytoplasm as an inactive heterodimer with its inhibitor protein subunit $(\mathrm{I} \kappa \mathrm{B})$. After stimulation, the $\mathrm{I} \kappa \mathrm{B}$ kinase induces the $\mathrm{I} \kappa \mathrm{B}$ phosphorylation, which promotes its degradation and causes the $\mathrm{NF}-\kappa \mathrm{B}$ complex to translocate into the nucleus (22). NF- $\kappa \mathrm{B}$ induces the expression of genes involved in key cellular processes including proliferation, survival, and differentiation (25).

PI3K is a major myogenic signaling molecule that also activates NADPH oxidase, which generates reactive oxygen species (ROS) and leads to NF- $\kappa \mathrm{B}$ activation. Many antioxidant enzymes are upregulated under conditions of oxidative stress. In contrast to other antioxidant enzymes, it is still unclear whether Prx2 is upregulated at the transcriptional level during oxidative stress. In this study, we hypothesized that Prx-2 is upregulated by the PI3K/NF- $\kappa \mathrm{B}$ pathway during muscle differentiation because ROS are more commonly generated during muscle differentiation than proliferation. Initially, we demonstrated that Prx-2 is induced via the PI3K/ $\mathrm{NF}-\kappa \mathrm{B}$ pathway during muscle differentiation and regeneration. We showed that Prx-2 plays an important role during myogenesis both in vitro and in vivo.

\section{Results}

\section{Prx-2 expression increases during muscle differentiation}

To observe the muscle differentiation phenotype in $\mathrm{C} 2 \mathrm{C} 12$ cells, we examined the morphological changes that occurred when the cells were cultured in differentiation medium (DM) for up to 96 hours. We also measured the fusion index to semiquantify the differentiation levels. As shown in Figure 1A, muscle differentiation progressed in a time-dependent manner. ROS levels were determined using dichlorofluorescein diacetate (DCFH-DA) after the cells were cultured in DM for 24 hours. ROS generation was significantly higher in cells cultured in DM than in proliferation medium (PM) (Fig. 1B,

FIG. 1. ROS generation and Prx-2 induction during muscle differentiation in C2C12 and primary myoblast cells. (A) Morphological changes and fusion index during the progression of differentiation in $\mathrm{C} 2 \mathrm{C} 12$ cells that were cultured in DM for up to $96 \mathrm{~h}$. The data shown are the means \pm SE of three independent experiments. ${ }^{*} p<0.005$ compared to cells cultured in PM. (B) Cellular ROS levels in C2C12 cell were estimated after the cells were cultured in DM for $24 \mathrm{~h}$ by measuring the DCF fluorescence and Amplex UltraRed assay. The data shown are the means \pm SE of three independent experiments. ${ }^{*} p<0.001$ when compared to cells cultured in PM. (C) The expression patterns of myogenin, MHC, Prx-2, and Prx-SO ${ }_{3} \mathrm{H}$ were determined under nonreducing [without $\beta$-mercaptoethanol, $(-) \beta$-mer] or reducing condition [with $\beta$-mercaptoethanol, $(+) \beta$ mer] conditions by Western blot analysis in C2C12 cells cultured in DM for up to 96h. D, dimeric form of Prx-2; M, monomeric form of Prx-2. Myogenin and MHC were used as muscle differentiation markers. The density values of Prx-2 relative to $\beta$-actin are expressed as the mean \pm SE of three independent experiments. ${ }^{*} * *<0.05$ when compared to the control values of the monomer and the dimer, respectively. (D) Cells cultured in PM or DM for $72 \mathrm{~h}$ were preincubated with $100 \mathrm{~m} M$ NEM for $15 \mathrm{~min}$ and were subsequently lysed in nonreducing SDS-sample buffer. Prx-2 expression in C2C12 cells was analyzed by Western blot analysis in C2C12 cells. The density values of Prx-2 relative to $\beta$-actin are expressed as the mean \pm SE of three independent experiments. ${ }^{*} \# p<0.05$ compared to the control values of the monomer and the dimer, respectively. (E) Morphological changes and the fusion index measured during the progression of differentiation in primary myoblast cells cultured in DM for $48 \mathrm{~h}$. The data shown are the means \pm SE of three independent experiments. ${ }^{*} p<0.001$ compared to cells cultured in PM. (F) Cellular ROS levels were estimated after primary myoblast cells were cultured in DM for 24 hours via DCF fluorescence and Amplex UltraRed assay in primary myoblast cells. The data are representative of at least three different experiments and are expressed as the means \pm SE. ${ }^{*} p<0.001$ compared to cells cultured in PM. (G) The expression patterns of myogenin, $\mathrm{MHC}$, Prx-2, and $\mathrm{Prx}-\mathrm{SO}_{3} \mathrm{H}$ in primary myoblast cells cultured in DM for up to $48 \mathrm{~h}$ were determined under $(-) \beta$-mer or $(+) \beta$-mer conditions by Western blot analysis. The density values of Prx- 2 relative to $\beta$-actin are expressed as the mean \pm SE of three independent experiments. ${ }^{*} * p<0.05$ compared to the control values of the monomer and the dimer, respectively. (H) Cells cultured in PM or DM for $48 \mathrm{~h}$ were preincubated with $100 \mathrm{mM}$ NEM for $15 \mathrm{~min}$ and were lysed in nonreducing SDS-sample buffer. Prx-2 expression in primary myoblast cells was analyzed by Western blot analysis. $\beta$-Actin was used as a loading control. The density values of Prx- 2 relative to $\beta$-actin are expressed as the mean \pm SE of three independent experiments. ${ }^{*}, p<0.05$ compared to the control values of the monomer and the dimer, respectively. All data are representative of at least three different experiments and are expressed as the means \pm SE. 
left). The semi-quantification of $\mathrm{H}_{2} \mathrm{O}_{2}$ generated during muscle differentiation was also performed using the Amplex UltraRed assay on the basis of a standard $\mathrm{H}_{2} \mathrm{O}_{2}$ dose curve. The results indicated that ROS generation was significantly higher in cells cultured in DM than in PM (Fig. 1B, right). The expression levels of muscle differentiation markers, such as myogenin and $\mathrm{MHC}$, were also increased during muscle differentiation (Fig. 1C). To determine whether Prx-2 expression was induced during the myogenic process, we observed the expression levels of Prx-2 under nonreducing or reducing conditions. Compared with cells cultured in PM, the expression levels of the dimeric and monomeric forms of Prx-2 were increased in a time-dependent manner in DM-cultured cells (Fig. 1C). To investigate further the oxidative state of Prx-2, we evaluated $\mathrm{Prx}-\mathrm{SO}_{3} \mathrm{H}$ levels by Western blot analysis and found that the hyperoxidized forms of Prxs were more abundant during differentiation (Fig. 1C). The same results were observed in $\mathrm{H} 9 \mathrm{c} 2$ rat cardiac myoblasts (data not shown). Next, we treated proliferating and differentiating cells with $100 \mathrm{mM}$ $N$-ethylmaleimide (NEM, a thiol-blocking agent) prior to lysis to prevent dimerization during the sample preparation. These samples were analyzed by Western blot analysis under nonreducing conditions. As shown in Figure 1D, higher expression levels of the monomeric and dimeric forms of Prx-2 were observed during muscle differentiation.

To verify the results observed in C2C12 cells, we isolated satellite cells from the mouse hind limb muscles, and monitored their differentiation by observing the morphological changes and measuring the fusion index. Primary cultured myoblasts were differentiated effectively in response to DM (Fig. 1E). $\mathrm{H}_{2} \mathrm{O}_{2}$ levels were significantly higher in cells cultured in DM rather than PM, which was in accordance to our observations in C2C12 cells (Fig. 1F). The expression levels of the dimeric and monomeric forms of Prx- 2 also increased in primary cultured myoblasts after differentiation was induced for 48 hours (Figs. $1 \mathrm{G}$ and $1 \mathrm{H}$ ). Therefore, we concluded that Prx-2 expression increases during muscle differentiation.

\section{Prx-2 is upregulated at the transcriptional level during muscle differentiation}

We hypothesized that the increase in Prx-2 expression may occur at the transcriptional level. To test this hypothesis, we performed semi-quantitative RT-PCR (Fig. 2A) and real-time quantitative RT-PCR (Fig. 2B). Both experiments revealed that Prx-2 mRNA level increased during muscle differentiation. To rule out the possibility of mRNA stabilization, we first induced the upregulation of Prx-2 mRNA by incubating the cells in DM for $24 \mathrm{~h}$ and subsequently treated cells with actinomycin D to inhibit de novo mRNA synthesis in PM or DM for up to $24 \mathrm{~h}$ to assess the mRNA decay rates by semiquantitative RT-PCR (Fig. 2C) and real-time quantitative RTPCR (Fig. 2D). We observed that the transcription of Prx-2 mRNA was completely inhibited $24 \mathrm{~h}$ after actinomycin D treatment. More importantly, the decay rate of Prx-2 mRNA was similar for the cells cultured in both PM and DM, indicating that the induction of Prx-2 expression during muscle differentiation is caused by transcriptional activation.

To determine whether the induction of Prx-2 during muscle differentiation is dependent on the synthesis of new proteins, we treated the cells with cycloheximide to inhibit protein synthesis in PM or DM for up to $24 \mathrm{~h}$ after induction of Prx-2
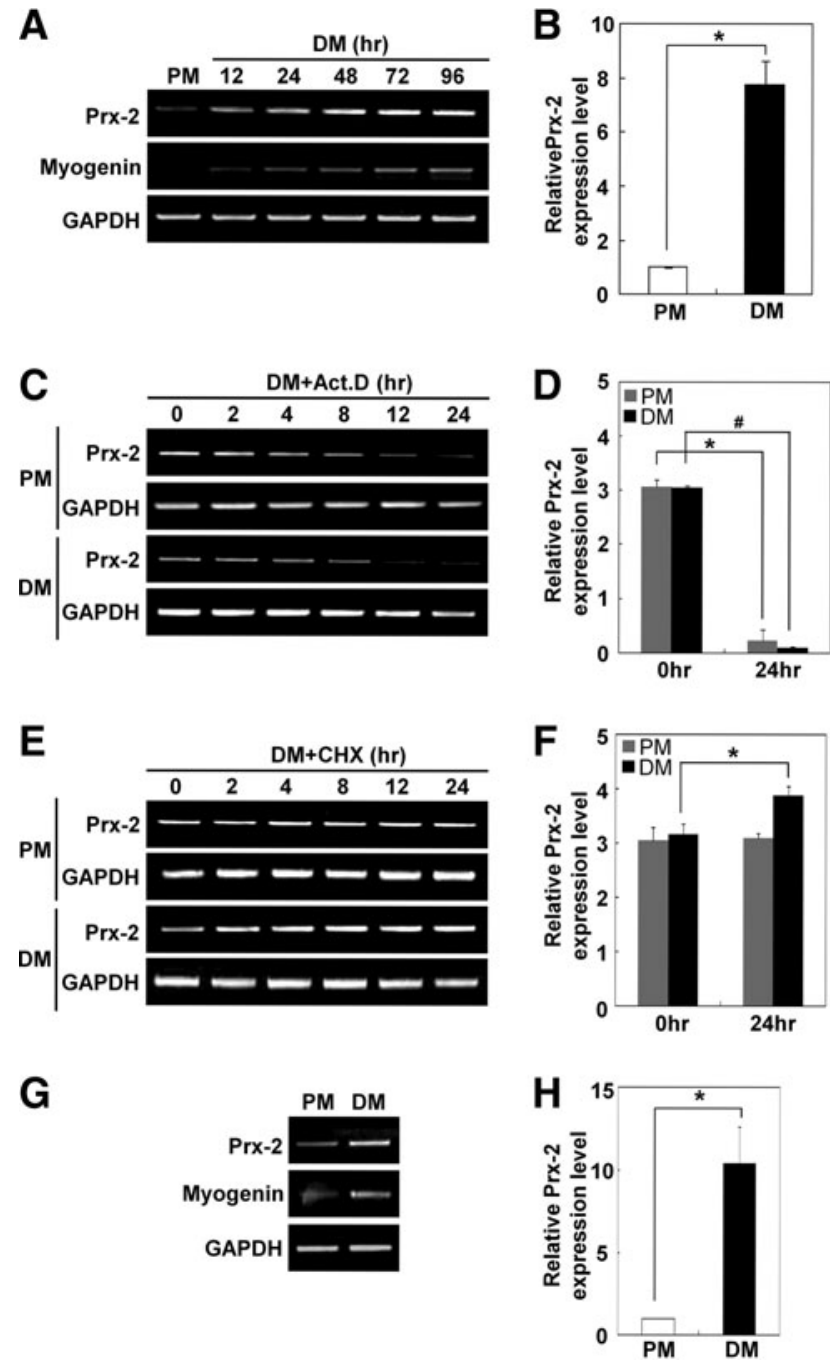

FIG. 2. The induction of Prx-2 mRNA during muscle differentiation in $\mathrm{C} 2 \mathrm{C} 12$ and primary myoblast cells. (A and B) Cells grown in PM were induced to differentiate for the indicated time periods by incubation in DM. Prx-2 and myogenin mRNA levels were analyzed by semi-quantitative RT-PCR (A). GAPDHmRNA was used as an internal control. Prx- 2 mRNA level was analyzed by real-time quantitative RT-PCR after $\mathrm{C} 2 \mathrm{C} 12$ cells were cultured in $\mathrm{DM}$ for $72 \mathrm{~h}$ (B). The data are representative of at least three different experiments and are expressed as the means $\pm \mathrm{SE}$. ${ }^{*} p<0.001$ compared to cells cultured in PM. (C and D) C2C12 cells incubated in DM for $24 \mathrm{~h}$ were again exposed to PM or DM with actinomycin D [Act.D $(5 \mu \mathrm{g} / \mathrm{mL})$ ], and Prx-2 mRNA levels were determined by semi-quantitative RT-PCR analysis $(C)$ and realtime quantitative RT-PCR analysis (D). The data shown are the means \pm SE of three independent experiments. ${ }^{*} \# p<0.001 \mathrm{com}-$ pared to untreated cells in PM and DM respectively. (E and F) C2C12 cells incubated in DM for $24 \mathrm{~h}$ were exposed to PM or DM with cycloheximide [CHX $(20 \mu \mathrm{g} / \mathrm{mL})]$, and semi-quantitative RT-PCR (E) and real-time quantitative RT-PCR (F) were conducted. The data are representative of at least three different experiments and are expressed as the means \pm SE. ${ }^{*} p<0.05$ compared to untreated cells in DM. (G and $\mathbf{H}$ ) Prx-2 mRNA level in primary myoblast cells was analyzed by semi-quantitative RTPCR (G) and real-time quantitative RT-PCR (H) after the cells were cultured in DM for $48 \mathrm{~h}$. The data shown are the means $\pm S E$ of three independent experiments. ${ }^{*}{ }^{*} p<0.001$ compared to cells cultured in PM. All data are representative of at least three different experiments and are expressed as the means \pm SE. 
under DM culture conditions for $24 \mathrm{~h}$. As shown in Figures $2 \mathrm{C}$ and 2D, cycloheximide did not block the increase in Prx-2 mRNA levels. Instead, cycloheximide increased Prx-2 mRNA levels in DM-cultured cells compared to PM-cultured cells as measured by semi-quantitative RT-PCR (Fig. 2E) and real-time quantitative RT-PCR (Fig. 2F). Using semi-quantitative RT-PCR (Fig. 2G) and real-time quantitative RT-PCR (Fig. 2H), we also found that Prx-2 mRNA level was upregulated at the transcriptional level during the differentiation of primary myoblasts. Taken together, these data suggested that Prx-2 is transcriptionally upregulated during muscle differentiation and that this mRNA induction is independent of new protein synthesis.

Upregulation of Prx-2 by $\mathrm{H}_{2} \mathrm{O}_{2}$ occurs only in myoblasts

We next investigated whether Prx-2 expression also increases in response to exogenous ROS as observed during muscle differentiation. For these experiments, we treated several cell lines with $\mathrm{H}_{2} \mathrm{O}_{2}$ for 6 hours. The appropriate concentrations of $\mathrm{H}_{2} \mathrm{O}_{2}$ were determined using MTT assay as shown in Supplementary Fig. S1 (Supplementary Data are available online at www .liebertonline/ars). Interestingly, the expression levels of the monomeric and dimeric forms of Prx-2 only increased in a concentration-dependent manner in C2C12 mouse myoblasts and $\mathrm{H} 9 \mathrm{c} 2$ rat cardiac myoblasts. The hyperoxidized forms of Prxs were also increased during differentiation. In contrast, the level of Prx-2 expression did not change in the NIH3T3, MEF, HEK293, and Chang cell lines; only the hyperoxidized forms of Prxs only were increased in these cell lines (Fig. 3A). To assess whether the $\mathrm{H}_{2} \mathrm{O}_{2}$-induced upregulation of Prx-2 expression in myoblasts occurs at the transcriptional level, we performed semi-quantitative RT-PCR and real time quantitative RT-PCR analysis under the same conditions as described for Figure 3A. As expected, an increase in Prx-2 mRNA levels was observed in myoblasts after $\mathrm{H}_{2} \mathrm{O}_{2}$ treatment (Figs. 3B and 3C).

\section{Antioxidants prevent the upregulation of Prx-2 and attenuate myogenesis}

To determine whether the ROS that are generated during muscle differentiation stimulate Prx-2 upregulation, we treated differentiating cells with $5 \mathrm{mMN}$-acetyl L-cysteine (NAC) and $5 \mathrm{mM}$ 4,5-dihydroxy-1,3-benzenedisulfonic acid (Tiron, a superoxide scavenger) for 24 hours and then measured ROS levels. As shown in Figure 4A, both antioxidants diminished the higher ROS levels in DM-cultured cells. They also both suppressed myotube formation, lowered the expression levels of myogenin, MHC, and Prx-2 (Figs. 4B and 4C), and attenuated both myogenesis and $\operatorname{Prx}-2$ induction in primary myoblasts as well (Figs, 4D and 4E). To further determine the expression levels of the dimeric and the monomeric forms of Prx-2 and Prx- $\mathrm{SO}_{3} \mathrm{H}$ in response to antioxidants, we analyzed their expression levels under nonreducing and reducing conditions (Supplementary Fig. S2).

\section{Transcriptional induction of Prx-2 is regulated by $N F-\kappa B$ during muscle differentiation}

To determine whether NF- $\kappa$ B regulates Prx-2 transcription during muscle differentiation, we analyzed Prx-2 promoter with the Genomatix program. As shown in a schematic representation of potential transcription factor binding sites (Fig.
5A), we found that Prx-2 promoter contains two putative NF$\kappa \mathrm{B}$ binding sites that are located between 3361 and $3352 \mathrm{bp}$ (candidate \#1) and between 3254 and 3245 bp (candidate \#2) upstream of Prx-2 start codon.

To test whether NF- $\kappa \mathrm{B}$ is required for Prx-2 transcription, we first constructed wild-type luciferase plasmids (W1-1097 and W2-736). The W1-1097 plasmid contained both putative $\mathrm{NF}-\kappa \mathrm{B}$ binding sites (candidates \#1 and \#2), while the W2-736 plasmid contained only one putative NF- $\kappa \mathrm{B}$ binding site (candidate \#2). We also made constructs containing either a point mutation each putative NF- $\kappa$ B binding site (M1-1097 and M2-736) or in both binding sites (M3-1097) to identify the functional NF- $\kappa$ B binding site on Prx-2 promoter. These resultant reporter plasmids were transiently introduced into C2C12 cells, and the activity of Prx-2 promoter activity was monitored by measuring luciferase activity during the differentiation process. The luciferase activity increased with the transfection of the -3697 to -2600 sequence of Prx-2 upstream promoter located upstream of the start codon. When the candidate site \#1 was mutated (M1-1097), the transcriptional activity of NF- $\kappa$ B was dramatically abolished. However, the mutation in candidate site \#2 (M2-736) did not change the level of luciferase activity from wild-type construct in the reporter assay. The double mutation constructs (M3-1097) also displayed basal levels of luciferase activity. We did not observe any luciferase activity in cells cultured under PM or DM conditions when empty pGL3-basic vector was used (Fig. 5B).

Next, we used oligonucleotides containing each putative $\mathrm{NF}-\kappa \mathrm{B}$ binding sites to perform an electrophoretic mobility shift assay (EMSA). Only the oligonucleotide containing candidate site \#1 showed strong binding, while the oligonucleotide containing candidate site \#2 did not show any significant binding (Fig. 5C). The binding was abolished by a hundred-fold excess amount of unlabeled competitor oligonucleotides. A Chromatin Immunoprecipitation (ChIP) assay also showed that candidate site \#1 is associated with NF- $\kappa \mathrm{B}$ (Fig. 5D). Therefore, we concluded that the NF- $\kappa \mathrm{B}$ binding site that is located between 3361 and 3352 bp upstream of Prx-2 start codon is indispensable for the induction of Prx-2 mRNA.

To further investigate the effect of the NF- $\kappa$ B on Prx-2 induction, we treated cells with NF- $\kappa$ B inhibitors PDTC (pyrrolidinedithiocarbamate acid) and parthenolide and observed the translocation of NF- $\kappa$ B (Fig. $5 \mathrm{E}$ ). As expected, NF- $\kappa \mathrm{B}$ p65 and NF- $\kappa$ B p50 were both translocated from the cytosol to the nucleus in cells cultured with DM, but NF- $\kappa$ B inhibitors prevented this nuclear translocation. We also observed that NF$\kappa \mathrm{B}$ inhibitors decreased the protein levels of Prx-2, myogenin, and $\mathrm{MHC}$, and inhibited myotube formation (Figs. 5F and $5 G)$. Also, siRNA knockdown of NF- $\kappa$ B p 65 produced the same results as with the inhibitors (Figs. 5H and 5I).

\section{Antioxidants prevent NF-KB from binding to Prx-2 promoter during muscle differentiation}

To further investigate whether NF- $\kappa \mathrm{B}$ binding to Prx -2 promoter was suppressed by antioxidants during muscle differentiation, we treated the differentiating cells with NAC and Tiron and then observed NF- $\kappa$ B activity. The luciferase activity of Prx-2 promoter was significantly reduced by antioxidants, compared with the untreated transfectants (Fig. 6A). Both antioxidants dramatically decreased NF- $\kappa$ B binding to Prx-2 promoter as determined by both the ChIP assay and the 
A
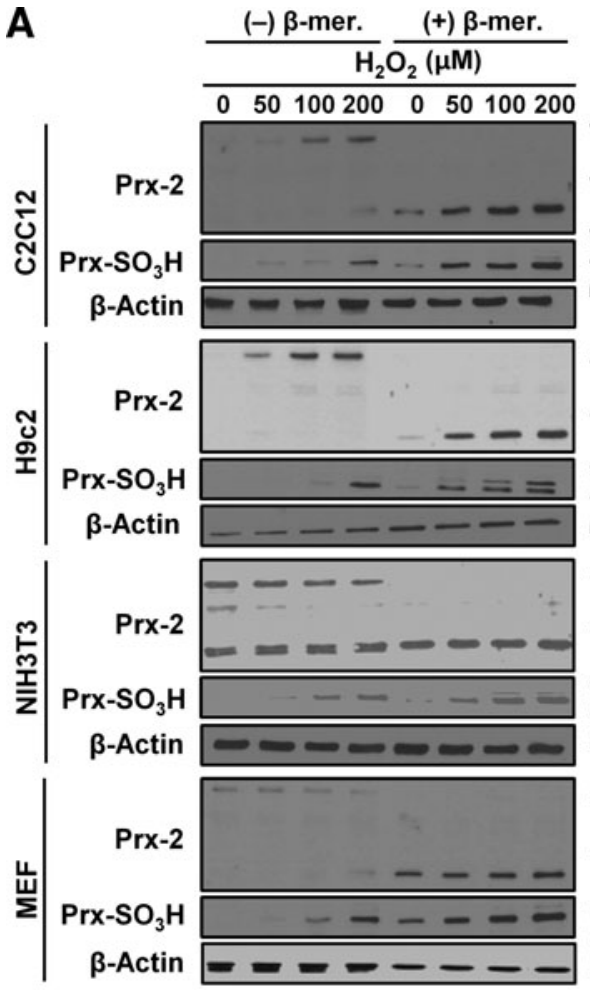

$\beta$-Actin
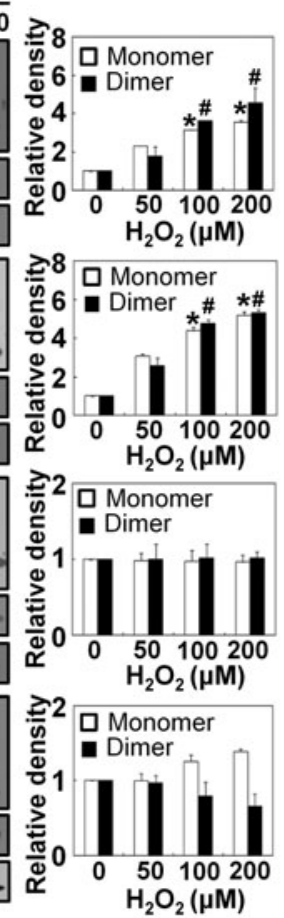

B
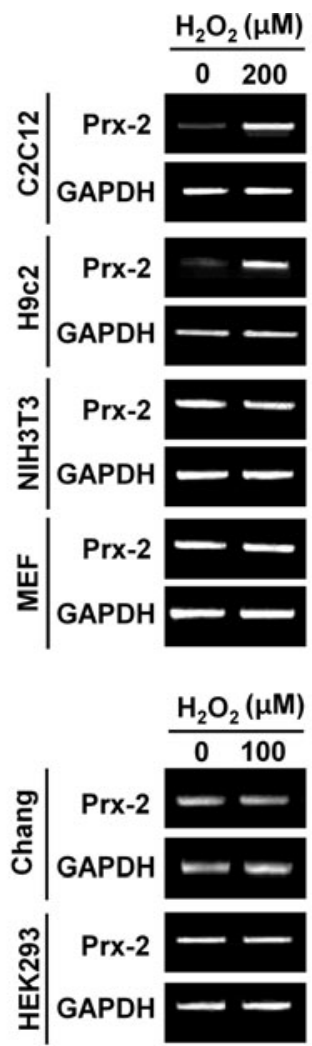
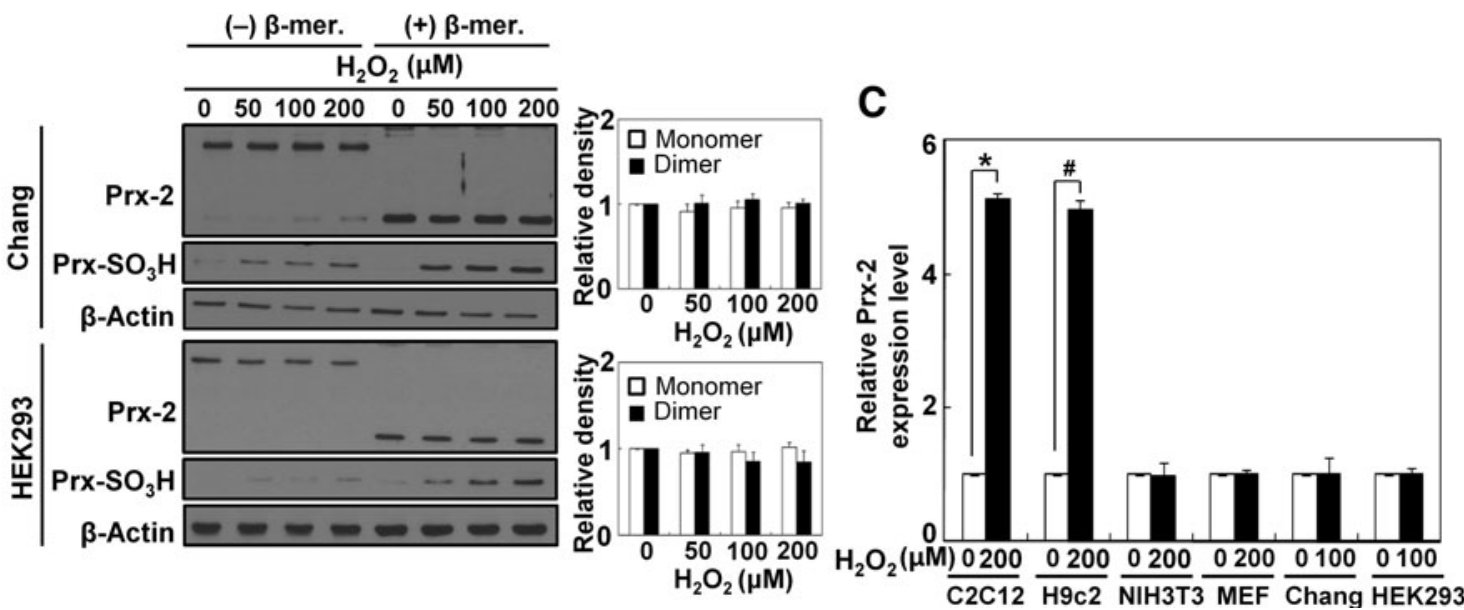

FIG. 3. Prx-2 regulation in response to $\mathrm{H}_{2} \mathrm{O}_{2}$. (A) $\mathrm{C} 2 \mathrm{C} 12$ cells were treated with various concentrations of $\mathrm{H}_{2} \mathrm{O}_{2}(0-200 \mu M)$ for $6 \mathrm{~h}$. The expression patterns of Prx-2 and Prx- $\mathrm{SO}_{3} \mathrm{H}$ were determined by Western blot analysis. $\beta$-Actin was used as a loading control. The density values of Prx-2 relative to $\beta$-actin are expressed as the mean \pm SE of three independent experiments. ${ }^{*} p<0.05$ compared to the control values of the monomer and the dimer, respectively. (B and C) Semi-quantitative RT-PCR (B) and real-time quantitative RT-PCR (C) were performed to analyze changes in the expression levels of Prx-2 mRNA after exposure to $\mathrm{H}_{2} \mathrm{O}_{2}$ for $6 \mathrm{~h}$. All data are representative of at least three different experiments and are expressed as the means \pm SE. ${ }^{*} \# p<0.001$ compared to untreated C2C12 and $\mathrm{H} 9 \mathrm{c} 2$ cells, respectively.

EMSA (Figs. 6B and 6C). In addition, they both prevented nuclear translocation of NF- $\kappa \mathrm{B}$ p 65 and NF- $\kappa \mathrm{B}$ p50 from the cytosol to the nucleus in cells cultured with DM (Fig. 6D).

\section{$P I 3 K$ is involved in $N F-\kappa B$ activation during muscle differentiation}

The PI3K signaling pathway is crucial for muscle differentiation (11). To examine whether the PI3K pathway is also involved in muscle differentiation, we treated the differentiating cells with LY294002 (a PI3K inhibitor) or LY303511 (a negative control for LY294002). In an effort to assess the effectiveness and specificity of LY294002, we monitored p-Akt level during muscle differentiation and observed that the level of p-Akt decreased when PI3K was inhibited (Fig. 7A). Previous reports have indicated that $\mathrm{NF}-\kappa \mathrm{B}$ can be activated downstream of PI3K during muscle differentiation $(11,28)$. Therefore, we tested whether inhibition of PI3K suppresses 
FIG. 4. Antioxidants prevent the upregulation of Prx-2 in C2C12 and primary myoblast cells. (A) Cellular ROS levels were estimated by measuring DCF fluorescence in the absence or presence of NAC or Tiron $(5 \mathrm{mM})$ after C2C12 cells were cultured in DM for $24 \mathrm{~h}$. The data are representative of at least three different experiments and are expressed as the means \pm SE. ${ }^{*} p<0.001$ compared to cells cultured in PM and ${ }^{\#} p<0.005$ compared to cells cultured in DM. (B) Morphological changes and the fusion index were measured in $\mathrm{C} 2 \mathrm{C} 12$ cells. The data are representative of at least three different experiments and are expressed as the means \pm SE. ${ }^{*} p<0.001$ compared to cells cultured in PM; ${ }^{*} p<0.005$ compared to cells cultured in DM. (C) Myogenin, MHC, and Prx-2 expression levels were determined by Western blot analysis after C2C12 cells were cultured in DM for $72 \mathrm{~h}$ in the absence or presence of NAC or Tiron $(5 \mathrm{mM})$. (D) Morphological changes and the fusion index were measured in primary myoblast cells. The data are representative of at least three different experiments and are expressed as the means $\pm \mathrm{SE}$. ${ }^{*} p<0.001$ compared to cells cultured in PM; ${ }^{*} p<0.005$ compared to cells cultured in DM. (E) Myogenin, MHC, and Prx-2 expression levels were determined by Western blot analysis after primary myoblasts were cultured in DM for $48 \mathrm{~h}$ in the absence or presence of NAC or Tiron $(2 \mathrm{mM})$ after. All data are representative of at least three different experiments and are expressed as the means $\pm \mathrm{SE}$. The band intensities shown in $\mathbf{C}$ and $\mathbf{E}$ are the average of three independent experiments.

NF $-\kappa \mathrm{B}$ activation and lower Prx-2 protein levels during muscle differentiation. As shown in Figure 7B, inhibiting PI3K prevented the nuclear translocation of NF- $\kappa \mathrm{B}$ p 65 and NF- $\kappa \mathrm{B}$ p50 in cells cultured with DM. Additionally, Prx-2 expression, which was regulated by NF- $\kappa \mathrm{B}$, was decreased in the presence of LY294002; this inhibitor also blocked muscle differentiation (Figs. 7C and 7D). As expected, LY303511 did not have the effects as LY294002. Therefore, we concluded that the PI3K/ NF $-\kappa \mathrm{B}$ signaling pathway performs a key function in the induction of Prx-2 expression during muscle differentiation.

\section{Prx-2 overexpression attenuates muscle differentiation}

To understand further the role of Prx-2 in myogenesis, we transfected $\mathrm{C} 2 \mathrm{C} 12$ cells with either an empty pCMV-Tag2B vector or the $\mathrm{PCMV}$-Tag2B-Prx-2 plasmid and then cultured the cells using DM. As shown in Figure 8A, Prx-2 was highly overexpressed after transient transfection with pCMV-Tag2BPrx-2, and Prx-2 overexpression attenuated myogenesis (Figs. $8 \mathrm{~A}$ and $8 \mathrm{~B})$. The levels of intracellular ROS were lower in
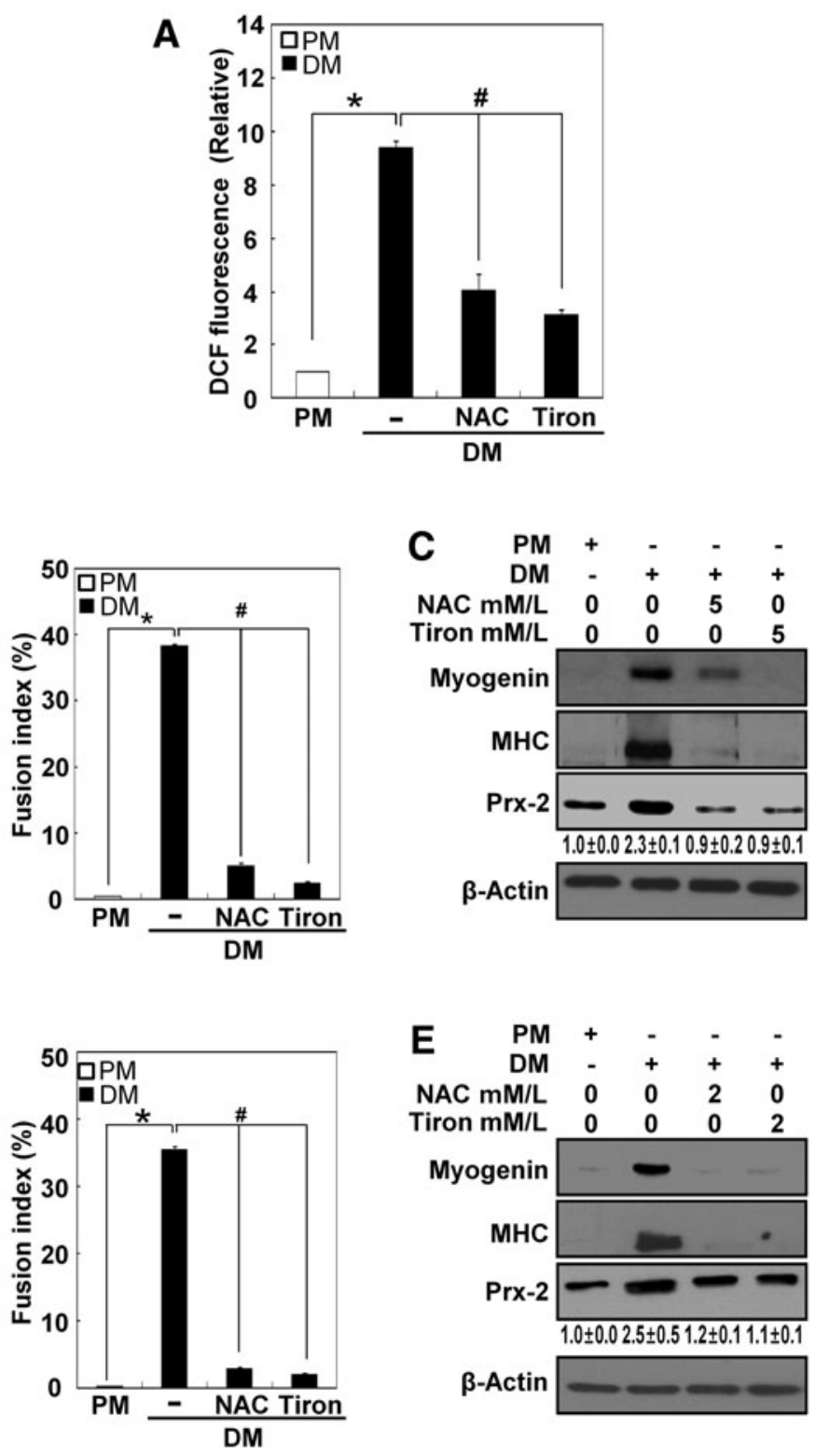

C2C12 cells transfected with pCMV-Tag2B-Prx-2 than in control pCMV-Tag2B-transfected cells (Fig. 8C). Collectively, our data showed that overexpression of Prx-2 attenuates myogenesis.

\section{Prx-2 knockdown upregulates the expression levels of other antioxidant enzymes}

To assess the effects of Prx-2 on muscle differentiation, we used Prx-2-specific siRNA to completely eliminate its expression (Fig. 9A). Inhibiting Prx-2 resulted in a more distinct myotube formation and an increase in the expression levels of both myogenin and MHC (Figs. 9A and 9B). The loss of Prx-2 also increased the expression levels of other antioxidant enzymes, including all other Prx family members, Trx1 and catalase, which are cyotosolic antioxidants (Fig. 9C). Moreover, the levels of intracellular ROS were higher in cells transfected with Prx-2 siRNA than in cells transfected with scrambled siRNA. The levels of intracellular ROS were significantly reduced after treatment with catalase (1000 U/ 
A

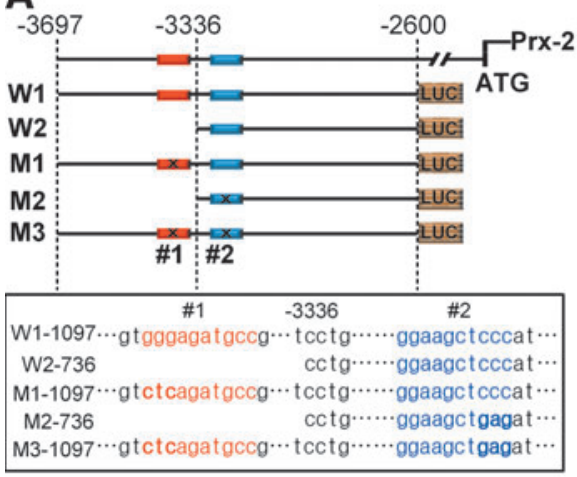

B

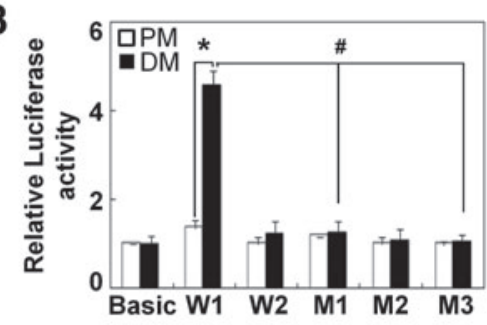

C

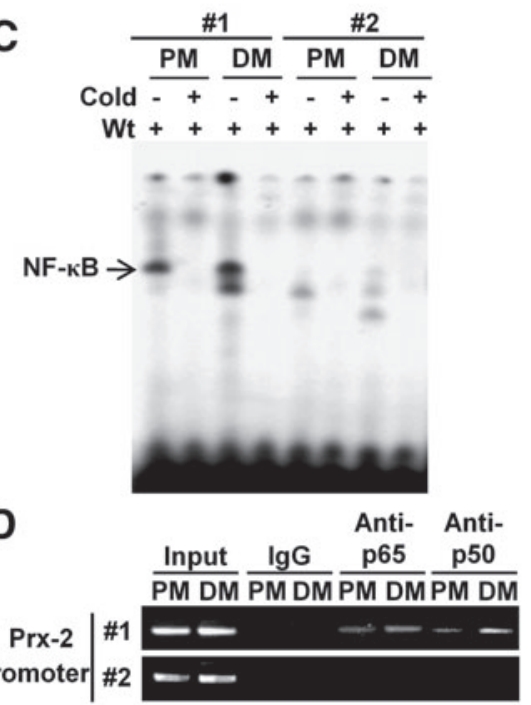

E

Cyt

F

G

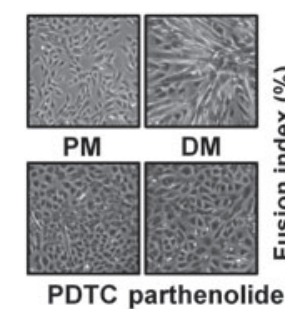

H

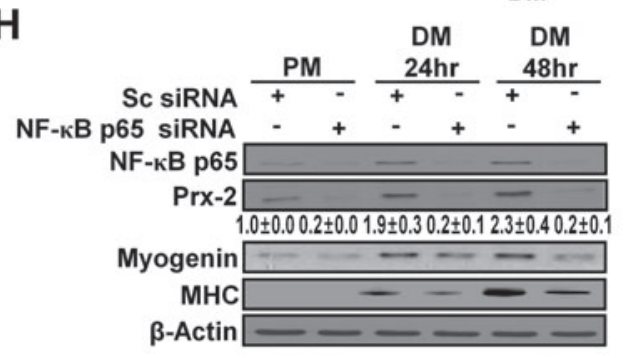

I

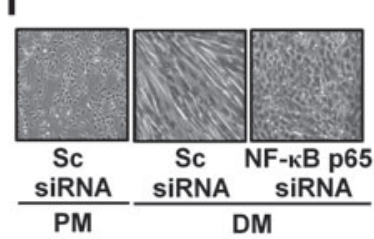

$\mathrm{PM}+-\cdot-$

$\mathrm{DM}-+++$

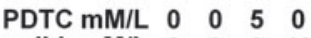

parthenolide $\mu \mathrm{M} / \mathrm{L} \quad \begin{array}{llll}0 & 0 & 0 & 10\end{array}$

NF кB p65 $=-\cdots$

NF-кB p50 - - -

Prx-2 ---$10 \pm 0.02400 .10990 .000 .940 .1$

$\beta$-Actin ---

Nucleus $\mid$\begin{tabular}{c|c|} 
NF-кB p65 & - \\
NF-кB p50 & - \\
LaminB & $-\infty--$
\end{tabular}

$\mathrm{PM}+\cdot-\cdot$

$\mathrm{DM}-+++$

PDTC $\mathrm{mM} / \mathrm{L} \quad 0 \quad 0 \quad 0 \quad 5 \quad 0$

parthenolide $\mu \mathrm{M} / \mathrm{L} \quad \begin{array}{llll}0 & 0 & 0 & 10\end{array}$

Myogenin

MHC

$\beta$-Actin $-6=$

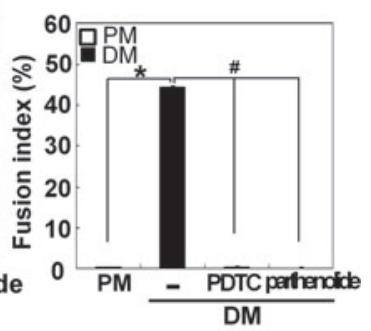

tion mixture. (D) ChIP assay. Chromatin from individual samples was precipitated using the indicated antibodies or with control IgG. Input genomic DNA (input) was used as a positive control and immunoprecipitation with nonspecific IgG (IgG) was used as a negative control. (E) $\mathrm{C} 2 \mathrm{C} 12$ cells were grown in DM for $72 \mathrm{~h}$ and exposed to the indicated doses of PDTC and parthenolide to inhibit NF- $\kappa$ B activity. The expression levels of NF- $\kappa \mathrm{B}$ p65, NF- $\kappa$ B p50, and Prx-2 were then determined by Western blot analysis. Lamin B was identified as a nuclear protein marker. (F and G) Differentiation was measured by Western

blot analysis (F), morphological changes, and measurement of the fusion index. The data are representative of at least three different experiments and are expressed as the means \pm SE. ${ }^{*} p<0.001$ compared to cells cultured in PM; ${ }^{*} p<0.001$ compared to cells cultured in DM (G). (H and I) C2C12 cells transfected with siRNA that is specific to NF- $\kappa$ B p65 were induced to differentiate in DM. The expression levels of NF- $\kappa$ B p 65 and Prx-2 were determined by Western blot analysis. Differentiation was measured by the expression levels of differentiation markers via Western blot analysis $\mathbf{( H )}$, morphological changes, and measurement of the fusion index. The data are representative of at least three different experiments and are expressed as the means \pm SE. ${ }^{*} p<0.005$ compared to cells transfected with scrambled siRNA that were grown in DM (I). All data are representative of at least three different experiments and are expressed as the means \pm SE. The band intensities shown in $\mathbf{E}$ and $\mathbf{H}$ are the average of three independent experiments.

$\mathrm{mL}$ ) in cells cultured with DM (Fig. 9D). In primary myoblasts, Prx-2 expression was also completely suppressed by treatment with Prx-2-specific siRNA (Fig. 9E). As observed using $\mathrm{C} 2 \mathrm{C} 12$ cells, the suppression of Prx-2 expression induced a more distinct myotube formation and an increase in the expression levels of myogenin, MHC and other antioxidant enzymes, including Prx family members, Trx-1, and catalase (Figs. 9F and 9G). In addition, the levels of intracellular ROS were higher in cells transfected with Prx-2 siRNA than in cells transfected with scrambled siRNA. The levels of 
FIG. 6. Antioxidants prevent NF-кB from binding to Prx-2 promoter during muscle differentiation in $\mathrm{C} 2 \mathrm{C} 12$ cells. (A) $\mathrm{C} 2 \mathrm{C} 12$ cells were transiently transfected with each luciferase reporter construct and the luciferase reporter assay was performed in the absence or presence of NAC or Tiron $(5 \mathrm{mM})$ after the cells were cultured in DM for $72 \mathrm{~h}$. The relative luciferase activities were expressed in comparison with the activity of pGL3Basic. The data shown are the means \pm SE of three independent experiments. ${ }^{*} p<0.001$ compared to pGL3-Prx-2/1107 (W1) in cells cultured in PM; $p<0.001$ compared to pGL3-Prx-2/1107 in cells cultured in DM. (B) An EMSA and (C) a ChIP assay were also performed. (D) The expression levels of NF- $\kappa \mathrm{B}$ p 65 and NF- $\kappa \mathrm{B}$ p50 were determined by Western blot analysis after the cells were cultured in $\mathrm{DM}$ for $72 \mathrm{~h}$ in the absence or presence of NAC or Tiron $(5 \mathrm{mM})$. Lamin B was identified as a nuclear protein marker. All data are representative of at least three different experiments and are expressed as the means $\pm \mathrm{SE}$.
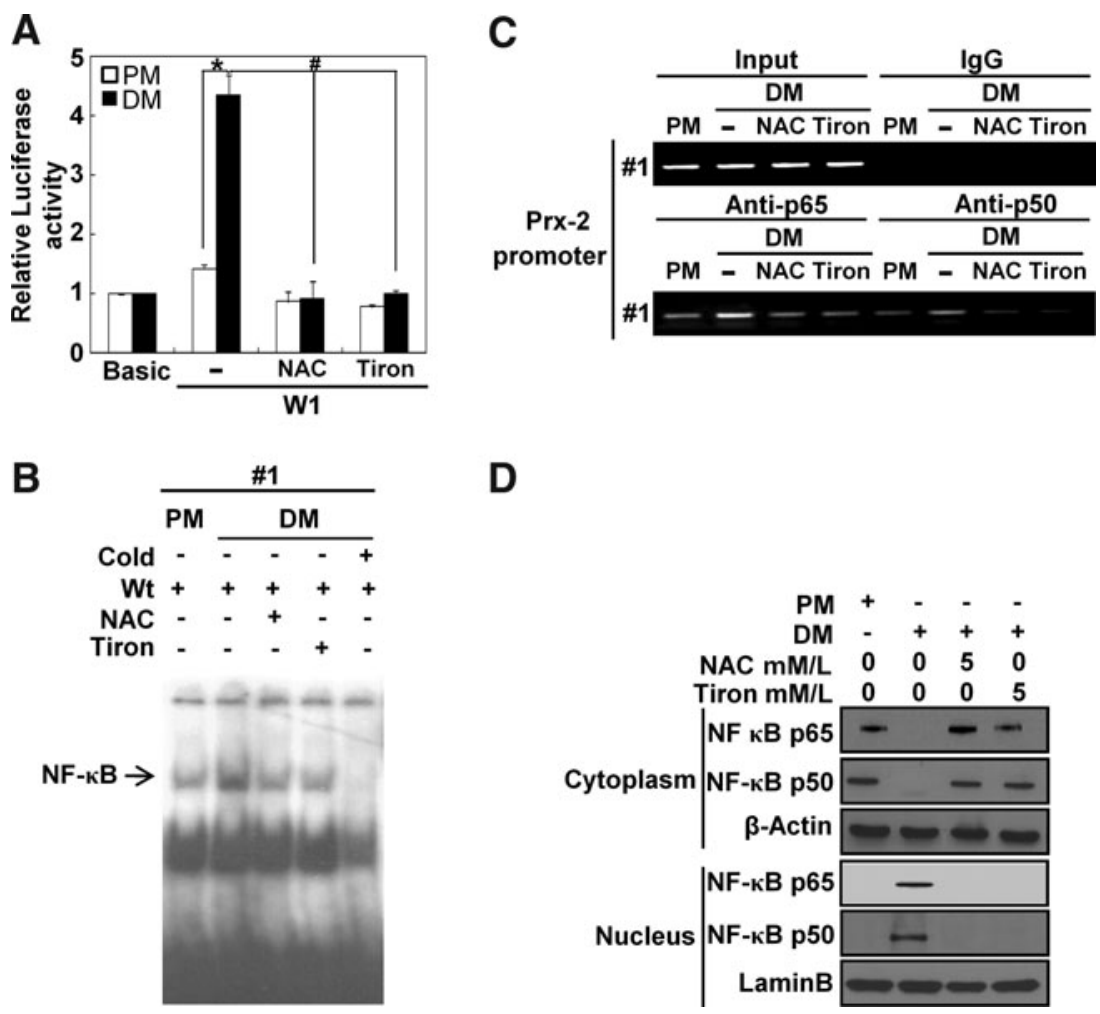

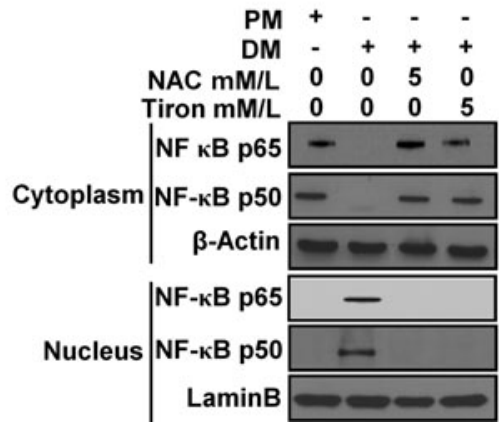

intracellular ROS were reduced by the addition of catalase to DM-cultured $\mathrm{C} 2 \mathrm{C} 12$ cells, as observed in C2C12 cells. In summary, we concluded that Prx-2 plays an important role in controlling the $\mathrm{H}_{2} \mathrm{O}_{2}$ that is generated during muscle differentiation.

\section{Prx-2 expression is upregulated in skeletal muscle upon cardiotoxin injury}

To determine whether Prx-2 participates in skeletal muscle differentiation in vivo, we examined its expression in a model of skeletal muscle regeneration. We triggered skeletal muscle injury by injecting cardiotoxin (CTX) into the tibialis anterior (TA) muscles. The tissues were analyzed at days 1, 3, and 5 $(n=3-5)$. As shown in Figure 10A, the expression levels of Prx2, myogenin, MyoD, and MHC were higher in CTX-injected TA muscles than in uninjured or PBS-injected TA muscles. Both semi-quantitative RT-PCR (Fig. 10B) and real-time quantitative RT-PCR (Fig. 10C) revealed that Prx-2 mRNA levels were remarkably upregulated after injury. The $\mathrm{H}$ and $\mathrm{E}$ stained cross-sections of TA muscle showed typical signs of injury and regeneration, 5 days after the CTX injection as indicated by the presence of newly formed myofibers with centralized nuclei. An immunohistochemical analysis showed that Prx-2, MyoD, and MHC were strongly expressed by muscle precursors and regenerating muscle fibers (Fig. 10D). In conclusion, Prx-2 expression is upregulated during injuryinduced muscle regeneration in vivo.

\section{Discussion}

In this study, we first noted that Prx-2 expression was upregulated by NF- $\kappa \mathrm{B}$ during muscle differentiation in $\mathrm{C} 2 \mathrm{C} 12$, $\mathrm{H} 9 \mathrm{c} 2$, and primary myoblast cells. We also found that Prx-2 was upregulated during the muscle regeneration process in vivo. In addition, the binding of NF- $\kappa \mathrm{B}$ to Prx-2 promoter was induced by the $\mathrm{H}_{2} \mathrm{O}_{2}$ that is generated during muscle differentiation. Prxs are present in all subcellular compartments and are expressed at various levels. The changes in the expression levels of the diverse family of Prxs have been extensively investigated in recent reports. For example, Prx-1 is the most widely studied and experiences the greatest transcriptional upregulation following treatment with a variety of factors that cause oxidative stress. NF-E2-related factor 2 (Nrf2) regulates of Prx-1 gene expression in response to electrophilic ROS-producing agents and hypoxia $(10,16)$. In addition, the stress-dependent induction of Prx gene expression has also been observed for other Prx family members. Previous studies have shown that Forkhead box O3 (FOXO3A) is required for the upregulation of Prx-3 under serum deprivation conditions in cardiac fibroblasts (2), and the Nrf2/Nrf1 pathway plays a role in the expression of Prx-3 and Prx-5 in response to quercetin (23). Also, Nrf2 is a key regulator of transcription of the Prx-6 gene under conditions of oxidative stress (4). However, the transcriptional regulatory mechanisms of Prx-2 have rarely been studied. A few reports have shown that Prx-2 is upregulated at the protein level during keratinocyte differentiation (36), and in response to cytokines or $\mathrm{H}_{2} \mathrm{O}_{2}$ in pancreatic $\beta$-cells (1). Therefore, we investigated Prx-2 promoter to identify a binding site for its transcription factor. We found that typical consensus NF- $\kappa$ B binding sequences exist in the human, rat, and mouse Prx-2 promoters. One of these, which was located between 3361 and $3352 \mathrm{bp}$ upstream of the mouse Prx-2 start codon, bound directly to NF- $\kappa$ B during muscle differentiation.

We also demonstrated that Prx-2 expression only increased in response to exogenous $\mathrm{H}_{2} \mathrm{O}_{2}$ in myoblasts and not in NIH3T3, MEF, Chang, and HEK293 cells. These data are supported by previous reports that demonstrated that only 

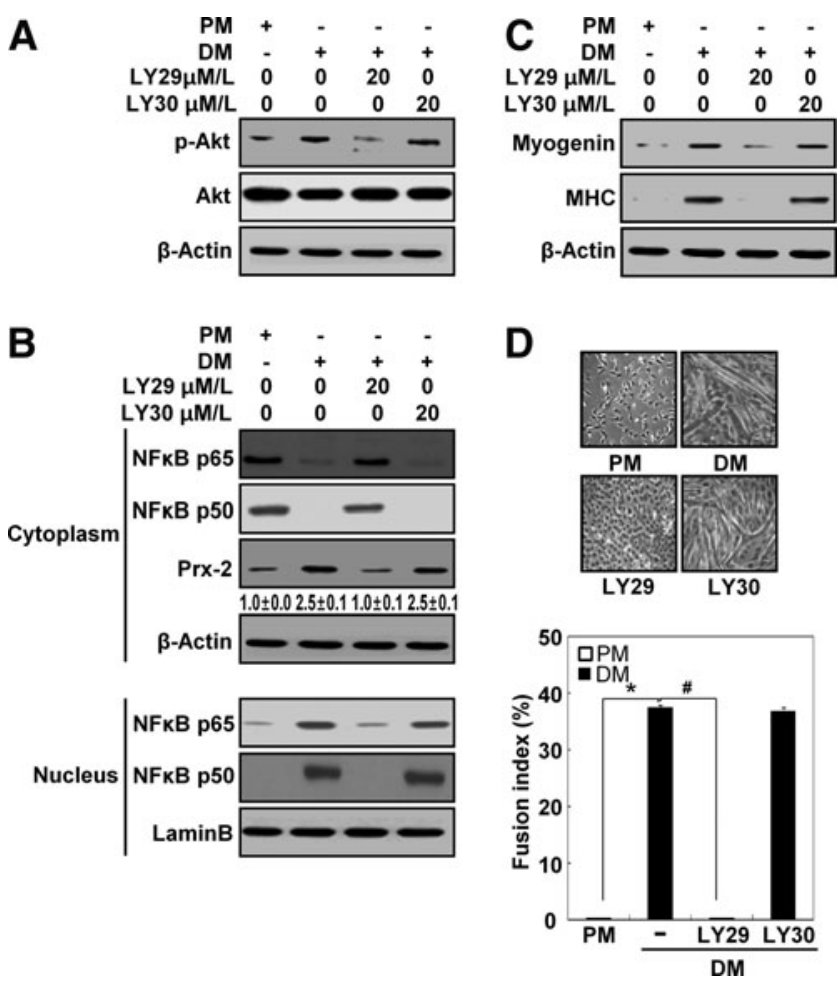

FIG. 7. The role of the PI3K/NF-кB pathway in Prx-2 upregulation during muscle differentiation in $\mathrm{C} 2 \mathrm{C} 12$ cells. (A) C2C12 cells were grown in DM for $72 \mathrm{~h}$ in the presence of LY294002 or LY303511 $(20 \mu M)$. To measure the effect of LY294002, the level of p-Akt was determined by Western blot analysis. (B) To test the effect of LY294002 on NF- $\kappa$ B activity, the nuclear translocation of NF- $\kappa \mathrm{B}$ p 65 and NF- $\kappa \mathrm{B}$ p50 was determined by Western blot analysis. Prx-2 expression levels were also determined by Western blot analysis in cells grown in DM for $72 \mathrm{~h}$ in the absence or presence of LY294002 (LY29) or LY303511 (LY30). The band intensities shown in (B) are average of three independent experiments. (C and D) Differentiation was assessed by measuring the expression levels of differentiation markers via Western blot analysis (C), morphological changes, and the fusion index. The data are representative of at least three different experiments and are expressed as the means \pm SE. ${ }^{*} p<0.001$ compared to cells cultured in PM; ${ }^{\#} p<0.001$ compared to cells cultured in DM (D). All data are representative of at least three different experiments and are expressed as the means \pm SE.

the hyperoxidized form of Prx-2 increases in response to $\mathrm{H}_{2} \mathrm{O}_{2}$ in the MEF and HEK293 cell lines $(3,15)$, while the total amount of Prx-2 remains the same. It is currently unclear why Prx-2 is not upregulated in the NIH3T3, MEF, HEK293, and Chang cell lines. Furthermore, we showed that NF- $\kappa \mathrm{B}$ was activated either by endogenous $\mathrm{H}_{2} \mathrm{O}_{2}$ generated during muscle differentiation or by exogenously added $\mathrm{H}_{2} \mathrm{O}_{2}$ and was involved in the upregulation of Prx-2 expression at the transcriptional level; this upregulation was also specific to myoblasts. Because the previous studies have shown that ROS-activated NF- $\kappa$ B promotes muscle differentiation and that its suppression blocks muscle differentiation and the transcriptional upregulation of MnSOD and iNOS $(28,32)$, it is likely that NF- $\kappa$ B plays diverse roles in muscle differentiation.

Myoblasts proliferate under high serum conditions, and confluent cells spontaneously differentiate after several days in
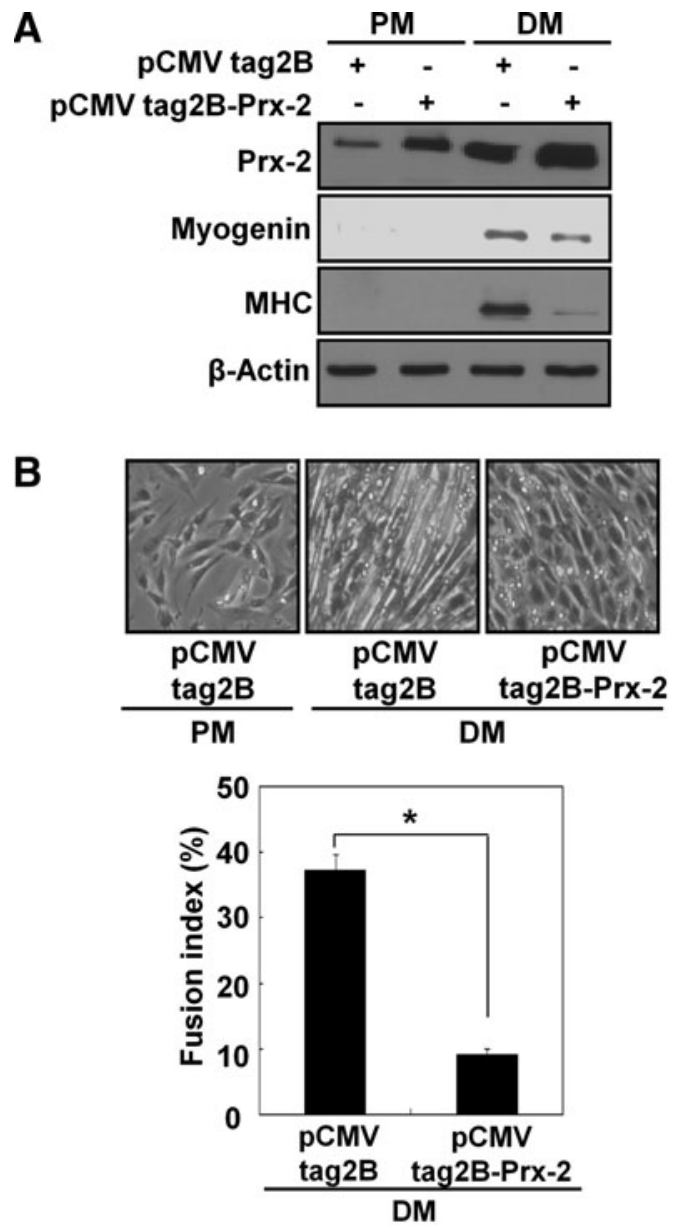

C

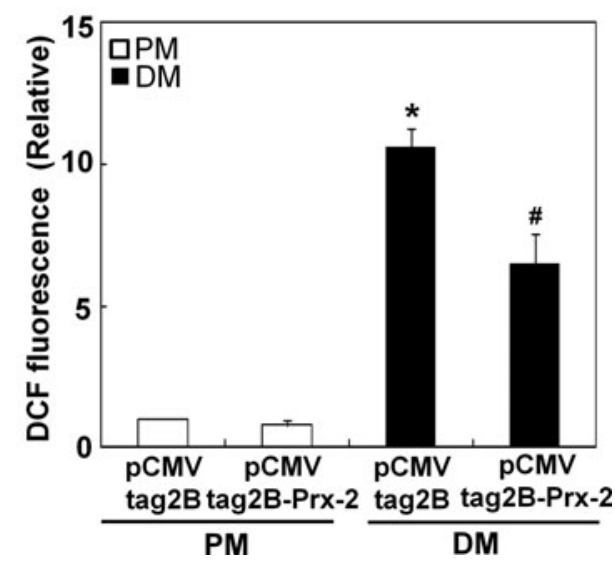

FIG. 8. Prx-2 overexpression attenuates myogenesis. (A) C2C12 cells were transiently transfected with either a control pCMV-tag2B or a pCMV-tag2B-Prx-2 vector. Prx2, myogenin, and MHC expression levels were determined by Western blot analysis after the cells were cultured for $72 \mathrm{~h}$ in DM. (B) Morphological changes and the fusion index were also measured. The data are representative of at least three different experiments and are expressed as the means $\pm \mathrm{SE}$. ${ }^{*} p<0.005$ compared to cells transfected with pCMV-tag2B and cultured in DM. (C) Cellular ROS levels were estimated by DCF fluorescence after $\mathrm{C} 2 \mathrm{C} 12$ cells were cultured in DM for $24 \mathrm{~h}$. The data are representative of at least three different experiments and are expressed as the means \pm SE. $* \# p<0.005$ compared to cells transfected with the control pCMV-tag2B vector and grown in PM and DM, respectively. 
A

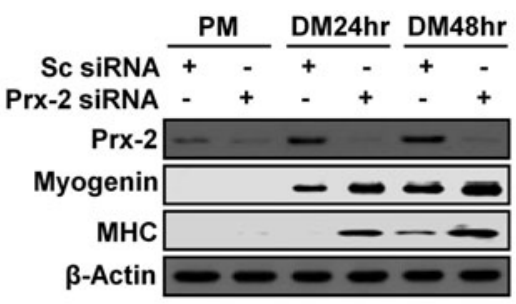

B

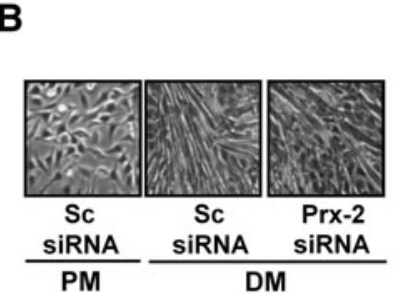

C

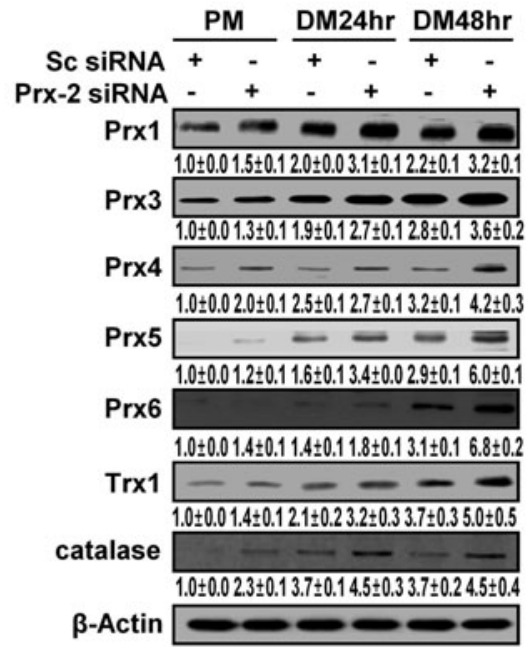

D

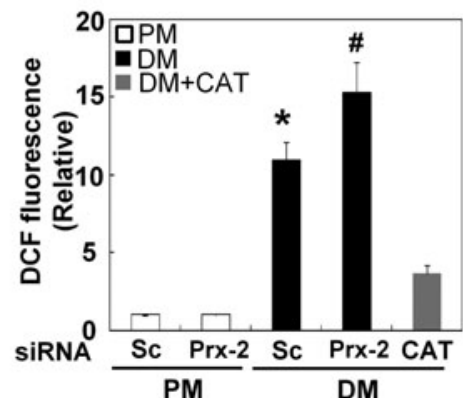

E

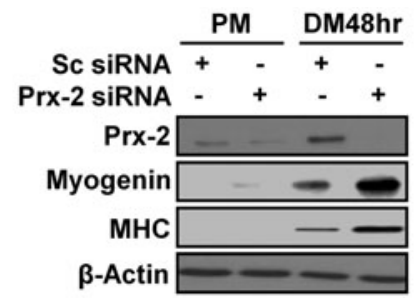

$\mathbf{F}$
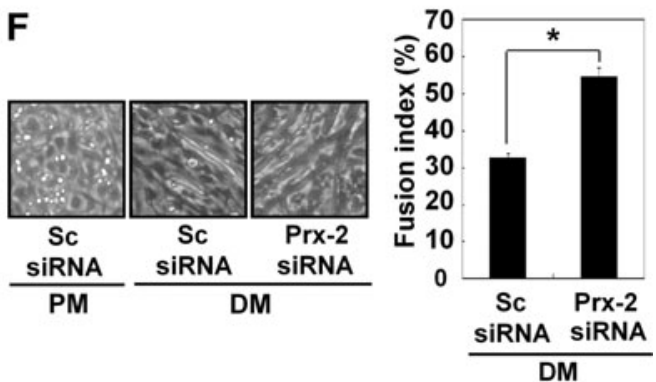

G
H

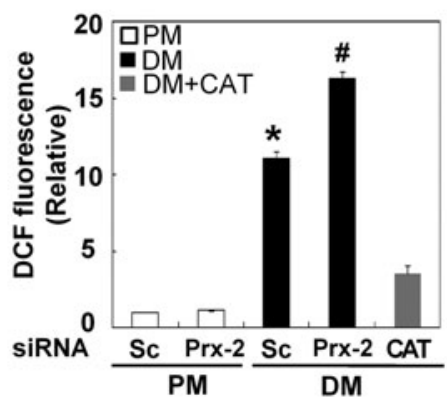

FIG. 9. Prx-2 knockdown upregulates the expression levels of other antioxidant enzymes in both $\mathrm{C} 2 \mathrm{C} 12$ and primary myoblast cells. (A) C2C12 cells transfected with Prx-2-specific siRNA (Prx-2 siRNA) were induced to differentiate in DM. Prx-2, myogenin, and MHC expression levels were determined by Western blot analysis. (B) Morphological changes and the fusion index were also measured. The data are representative of at least three different experiments and are expressed as the means \pm SE. ${ }^{*} p<0.005$ compared to cells transfected with scrambled siRNA (Sc siRNA) that were grown in DM. (C) The expression levels of Prx1-6, Trx-1, and catalase (CAT) were determined by Western blot analysis. (D) Cellular ROS levels were estimated by measuring DCF fluorescence after the cells were cultured in DM for $24 \mathrm{~h}$. Scrambled siRNA was used as a negative control for the knockdown. The data are representative of at least three different experiments and are expressed as the means \pm SE. ${ }^{*}, \#<0.001$ compared to cells transfected with scrambled siRNA and grown in PM and DM, respectively. (E) Primary myoblasts transfected with Prx-2-specific siRNA were induced to differentiate in DM. Prx-2, myogenin, and MHC expression levels were determined by Western blot analysis. (F) Morphological changes and a fusion index were also measured. The data are representative of at least three different experiments and are expressed as the means \pm SE. ${ }^{*} p<0.005$ compared to cells transfected with scrambled siRNA and grown in DM. (G) The expression levels of Prx1-6, Trx-1, and catalase were determined by Western blot analysis. (H) Cellular ROS levels were estimated by measuring DCF fluorescence after the cells were cultured in DM for $24 \mathrm{~h}$. The data are representative of at least three different experiments and are expressed as the means \pm SE. ${ }^{*} * 0<0.001$ compared to cells transfected with scrambled siRNA in PM and DM, respectively. The band intensities shown in $\mathbf{C}$ and $\mathbf{G}$ are the average of three independent experiments. 
A

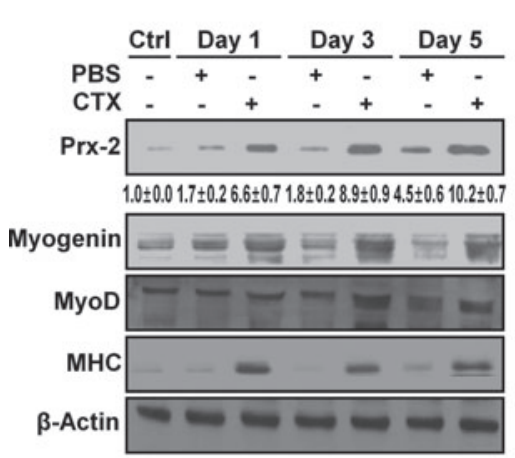

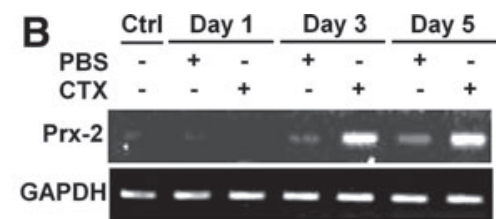

C

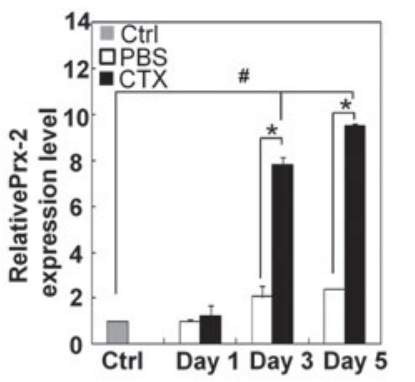

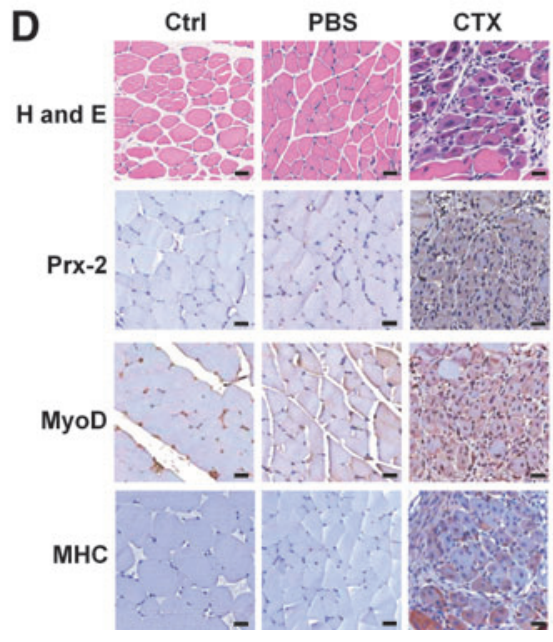

FIG. 10. Prx-2 expression after CTX injection. (A) Prx-2, myogenin, and MHC expression levels in TA muscles were determined by Western blot analysis at the indicated number of days after the injection of CTX or PBS. PBSinjected and uninjected TA muscles were used as controls. The band intensities shown in (A) are the average of three independent experiments. (B and C) The post-injection Prx-2 mRNA level was analyzed by semi-quantitative RT-PCR (B) and real-time quantitative RTPCR after injection (C). GAPDH mRNA was used as an internal control. The data are representative of at least three different experiments and are expressed as the means \pm SE. *,\# $p<0.001$ compared to PBS-injected and uninjected TA muscles, respectively. (D) TA muscles at 5 days after injection were stained with $\mathrm{H}$ and $\mathrm{E}$ and antibodies against Prx-2, MyoD, and MHC. MyoD and MHC were used as regeneration markers. Scale bars: $100 \mu \mathrm{m}$. low serum due to the autocrine expression of IGF acting through the IGF receptor (6). Moreover, IGF-1 and -2 stimulate muscle differentiation by activating several signaling pathways. These IGFs stimulate the myogenic process via PI3K. Previous reports have demonstrated that PI3K leads to the activation of NADPH oxidase, which, in turn, produces ROS during muscle differentiation $(17,28)$. In addition, NF- $\kappa$ B is a target for NADPH oxidase or mitochondria-derived ROS in skeletal myoblasts $(5,18,28)$. In this study, we showed that the inhibition of PI3K blocked NF- $\kappa \mathrm{B}$ activation, suppressed the expression of Prx-2, and impaired muscle differentiation. Therefore, we concluded that Prx-2 upregulation was induced by $\mathrm{NF}-\kappa \mathrm{B}$, which is downstream of the PI3K pathway during muscle differentiation. The scheme presented in Supplementary Fig. S3 depicts how Prx-2 is upregulated in the PI $3 \mathrm{~K} / \mathrm{NF}-\kappa \mathrm{B}-$ dependent myogenic signaling pathway.

The overexpression of Prx-2 prevented both myogenesis and ROS production. In contrast, Prx-2 knockdown improved muscle differentiation and upregulated the expression of other antioxidant enzymes, including all other Prx family members, Trx1, and catalase. Furthermore, intracellular ROS levels were higher in cells transfected with Prx-2-siRNA than in cells transfected with scrambled siRNA, suggesting that other antioxidant enzymes may compensate for the loss of Prx-2. These findings are supported by previous reports indicating that the expression of Prx-3 was increased in Prx-5-knockdown mice under conditions of hypoxia, suggesting that the upregulation of Prx-3 compensates for the knockdown of Prx5 (35). Also, these previous reports have shown that excessive $\mathrm{H}_{2} \mathrm{O}_{2}$ not only causes cell death but also impairs myogenesis, while physiological levels of $\mathrm{H}_{2} \mathrm{O}_{2}$ are a major signaling mediator for muscle differentiation $(7,19)$. Therefore, the enhanced levels of $\mathrm{H}_{2} \mathrm{O}_{2}$ following treatment with Prx-2-siRNA may accelerate myogenesis instead of impairing it. Our data showed that although Prx-2 is dispensable due to the compensatory upregulation of other antioxidant enzymes, it is an important antioxidant enzyme that is used to control excessive ROS during muscle differentiation.

In summary, we have provided novel evidence that Prx-2 is upregulated at the transcriptional level via the NF- $\kappa \mathrm{B} / \mathrm{PI} 3 \mathrm{~K}$ pathway during muscle differentiation. Also, Prx-2 knockdown induced the compensatory expressions of other antioxidant enzymes, including all other Prx family members, Trx1, and catalase, but it also increased intracellular ROS levels. Taken together, these results suggest that Prx-2 is an important molecule for the control of ROS homeostasis in myoblasts. It remains unclear why Prx-2 is upregulated only in myoblasts and not in other cell lines. It is possible that one or more myogenic transcription factors may cooperate with NF- $\kappa$ B to transcriptionally upregulate Prx-2 during muscle differentiation. 


\section{Materials and Methods}

\section{Materials}

Dulbeco's modified Eagle's Medium (DMEM), Dulbecco's modified Eagle's medium/F-12 (DMEM/F-12), fetal bovine serum (FBS), and calf serum were purchased from GIBCOBRL (Grand Island, NY). 2'7'-dichlorofluorescein diacetate (DCF-DA), actinomycin D, cycloheximide, pyrrolidine dithiocarbamate acid (PDTC), parthenolide, $\mathrm{H}_{2} \mathrm{O}_{2}$ solution, catalase, $\beta$-mercaptoethanol, and collagenase type I were acquired from Sigma (St. Louis, MO). Basic fibroblast growth factor was purchased from R \& D systems (Minneapolis, MN). LY294002 and LY303511 were obtained from Tocris Cookson, Ltd (Bristol, UK). $\left[\gamma^{32} \mathrm{P}\right]$ ATP was purchased from Amersham Biosciences (Little Chalfont, UK). N-ethylmaleimide (NEM), NF- $\kappa$ B probe for electrophoretic mobility shift assays, and antibodies specific to MHC, myogenin, Prx-1, NF$\kappa \mathrm{B}$ p50, Akt, and lamin B were purchased from Santa Cruz Biotechnology (Santa Cruz, CA). The antibodies specific to Prx 2-6, Prx- $\mathrm{SO}_{3} \mathrm{H}$ and Trx1 were obtained from Ab Frontier (Seoul, Korea). The antibody specific to catalase was acquired from Calbiochem (Darmstadt, Germany). The antibody specific to NF- $\kappa$ B p 65 was provided by Stressgen Biotechnologies (San Diego, CA). The antibody specific to $\beta$-actin was purchased from Abcam (Cambridge, MA). The antibody specific to phospho-Akt (S473) was acquired from Cell Signaling Technology (Beverly, MA).

\section{Satellite cell isolation}

C57BL/ 6 female mice were purchased from the Central Lab Animal Inc. (Seoul, Korea) and allowed to acclimate for a week before being used in the study. Animal experimentations were conducted according to the protocol approved by the Institutional Animal Care and Use Committee of Kyung Hee University. Hind limb muscles were surgically removed from 6-8-week-old C57BL/ 6 female mice, washed three times in Dulbecco's PBS, chopped in $0.025 \%$ of collagenase type I with fine scissors, incubated at $37^{\circ} \mathrm{C}$ for $90 \mathrm{~min}$ with stirring, and centrifuged at $350 \mathrm{~g}$ for $5 \mathrm{~min}$. The pellet was resuspended in $10 \mathrm{~mL}$ of complete medium (Ham's F10 supplemented with $20 \% \mathrm{FBS}$ and $1 \%$ penicillin-streptomycin), and the suspension was passed through a $100 \mu \mathrm{m}$ filter to remove muscle debris. Cells were centrifuged at $350 \mathrm{~g}$ for $5 \mathrm{~min}$, resuspended in complete medium, and seeded into $100-\mathrm{mm}$ gelatin-coated dishes. Basic fibroblast growth factor was added twice a day at a concentration of $4 \mathrm{ng} / \mathrm{mL}$. After $48 \mathrm{~h}$, cells were transferred to noncoated plates and incubated for $45-60 \mathrm{~min}$ to allow the fibroblasts to attach to plates. The floating cells were resuspended in complete medium (Ham's F10 supplemented with $2 \%$ FBS, 5\% Ultroser-G. and 1\% penicillin-streptomycin) containing $4 \mathrm{ng} / \mathrm{mL}$ of basic fibroblast growth factor, and then seeded into gelatin-coated dishes. When cells were confluent, they were placed in alpha minimum essential medium supplemented with $2 \%$ horse serum and $1 \%$ penicillinstreptomycin for differentiation.

\section{Cell culture}

C2C12 mouse myoblasts were grown in DMEM supplemented with 10\% FBS (proliferation medium, PM). Cells were induced to differentiate by placing them in DMEM containing $1 \%(\mathrm{v} / \mathrm{v})$ FBS (differentiation medium, DM), and full dif- ferentiation was achieved 6 days after induction of differentiation. Differentiation was evaluated on the basis of the morphological appearance of cells, fusion index, and the expression levels of the differentiation markers such as myogenin and MHC. NIH3T3, mouse embryonic fibroblasts (MEF), Chang human liver, and human embryonic kidney (HEK) 293 cell lines were maintained in PM. H9c2 rat cardiac myoblasts were grown in DMEM/F-12 containing 10\% (v/v) donor calf serum. Unless specified, the culture medium was replaced with fresh medium every 2 days.

\section{Measurement of fusion index}

Fusion index was determined as the ratio of the nuclei number in cells containing two or more nuclei to the total nuclei number in hematoxylin-stained $\mathrm{C} 2 \mathrm{C} 12$ and satellite myotubes. These cells were washed in PBS, fixed with $10 \%$ formaldehyde in PBS for $10 \mathrm{~min}$, and permeabilized with $0.5 \%$ Triton-X-100 in PBS for $5 \mathrm{~min}$. Next, they were stained with $10 \%$ hematoxylin solution for $5 \mathrm{~min}$ and then washed in distilled water. The number of nuclei was measured at least from the six different fields observed under microscope. Fusion $(\%)=($ number of nuclei in myotubes) / (total number of nuclei in myoblasts and myotubes) X 100.

\section{Cardiotoxin-induced muscle injury and regeneration}

A $10 \mu \mathrm{M}$ solution of cardiotoxin from Naja mossambica mossambica was injected into left tibialis anterior (TA) muscles of 6-8- week-old C57BL/ 6 female mice at $2 \mu \mathrm{L} / \mathrm{g}$ body weight using an insulin syringe. The equal volume of PBS was injected into right TA muscles. The uninjected TA muscles served as the control. After 1, 3, and 5 days, animals were euthanized, and the TA muscle was removed for analysis.

\section{Histology and immunohistochemistry}

Paraffin sections ( $5 \mu \mathrm{m}$ ) were prepared for hematoxylin and eosin ( $\mathrm{H}$ and $\mathrm{E})$ staining or immunohistochemical analysis. Paraffin sections were deparaffinized with xylenes and hydrated with a series of ethanol. Endogenous peroxidases were quenched with a short treatment of $1 \%$ hydrogen peroxide. To enhance the signal, we used antigen retrieval in citrate buffer and signal amplification with biotinylated tyramide. The deparaffinized and rehydrated specimens were incubated overnight at $4^{\circ} \mathrm{C}$ with antibodies against Prx-2 (1:200; Ab Frontier, Seoul, Korea), MyoD (1:50; Santa Cruz Biotechnology), and MHC (1:50; Developmental Study Hybridoma Bank, IA). The immunostained section was visualized with an EnVision Detection Kit (Dako, CA). Routine H and E-stained sections were examined to ensure the structural integrity of the tissues.

\section{Measurement of reactive oxygen species}

Intracellular ROS levels were measured with DCF-DA using FACS analysis as previously described (7). Cells were loaded with $20 \mu \mathrm{M}$ DCF-DA, and fluorescence was measured with a flow cytometer (FACSCalibur, Becton-Dickinson, Franklin Lakes, NJ). The mean DCF fluorescence intensity was measured with excitation at $488 \mathrm{~nm}$ and emission at $525 \mathrm{~nm}$.

Intracellular ROS was also measured by Amplex UltraRed assay as previously described (24). Briefly, cells were cultured 
in PM or DM, and washed twice with Krebs-Ringer phosphate buffer and incubated with $5 \mu M$ Amplex UltraRed reagent (Molecular Probes, Eugene, OR) and 0.1 unit $/ \mathrm{mL}$ horseradish peroxidase type II (Sigma, St. Louis, MO) in Krebs-Ringer phosphate (Sigma) for $40 \mathrm{~min}$. To identify the hydrogen peroxide-specific signal, control samples were co-incubated with $500 \mathrm{U} / \mathrm{mL}$ catalase. Triplicate readings were taken in a 96-well plate using $100 \mu \mathrm{L}$ samples of medium, and fluorescence was detected via a plate reader at excitation and emission of 530 and $580 \mathrm{~nm}$, respectively. $\mathrm{H}_{2} \mathrm{O}_{2}$ levels were calculated in terms of catalase-inhibitable signal and were normalized to cellular protein as measured by the Bio- $\mathrm{Rad} \mathrm{DC}$ assay. $\mathrm{H}_{2} \mathrm{O}_{2}$ concentration was estimated using a standard curve.

\section{Reverse transcription-PCR (RT-PCR)}

For the analysis of Prx-2 mRNA, total RNA was prepared using the TRIzol reagent (Invitrogen, San Diego, CA), and total RNA $(2 \mu \mathrm{g})$ was amplified by a two-step protocol using AMV reverse-transcriptase (Promega) and Taq polymerase. The PCR products were amplified using the following specific primers:

Prx-2 forward, 5'-GACTACAGAGGGAAGTACGTGG-3'; Prx-2 reverse, 5'-TCAGTTGTGTTTGGAGAAGTATTCC-3'; myogenin forward, 5'-ATGGAGCTGTATGAAACATCC CCC-3';

myogenin reverse, 5'-TTCCCTTGCTGGGCTGGGTGT TAG-3';

GAPDH forward, 5'-CAACTTTGGCATTGTGGAAGGG-3'; and GAPDH reverse 5'-ACACATTGGGGGTAGGAACA-3'.

The amplified products were visualized on a $1 \%$ agarose gel, and the amplified GAPDH fragment was used as an internal control for RT-PCR.

\section{Real-time quantitative RT-PCR}

The mRNA levels for Prx-2 and GAPDH were measured using SYBR ${ }^{\circledR}$ Green PCR Master Mix (Applied Biosystems, Foster City, CA) and the ABI PRISM ${ }^{\circledR} 7300$ real-time PCR system (Applied Biosystems) according to the manufacturer's instructions, after primer sequences were designed with the Primer 3 (Rozen and Skaletsky 2000; http://frodo.wi.mit .edu/, accessed June 22, 2009) and UCSC In-Silico PCR to select primer pairs that produced a unique PCR product.

Prx-2 mouse forward, 5'-CACGGAGATCATCGCTTTT AGC-3';

Prx-2 mouse reverse, 5'-TCAACACGCCGTAATTGTGT GAC-3';

GAPDH mouse forward, 5'-CAACTTTGGCATTGTGGAA GGG-3';

GAPDH mouse reverse 5'-ACACATTGGGGGTAGGA ACA-3';

Prx-2 rat forward, 5'-CACCTGGCCTGGATCAATAC-3';

Prx-2 rat reverse, 5'

GAPDH rat forward, 5'-CATGTTTGTGATGGGTGTGA-3';

GAPDH rat reverse, 5'-TCCACAGTCTTCTGAGTGGC-3';

Prx-2 human forward, 5'-CGTCTCGGTGGACTCTCAGT-3';

Prx-2 human reverse, 5'-ATAAAGAGGCCCCTGTAGGC-3'; GAPDH human forward, 5'-TGACCACAGTCCATGC CAT-3';

and GAPDH human reverse, $5^{\prime}$-TTCTAGACGGCAGGTC AGGT-3'.
Calculations were based on the "Delta-Delta method" using the following equation: $R$ (ratio) $=2^{-[\Delta C T \text { sample }-\Delta C T \text { control }]}$ (21). The data were expressed as fold changes of the treatment groups relative to the control. GAPDH was amplified as a control for real-time quantitative RT-PCR.

\section{Construction of plasmids for Prx-2 promoter analysis}

The Prx-2 promoter sequence was analyzed using the MatInspector of Genomatrix (http://www.genomatix.de./, accessed Sep. 16, 2009). For the construction of the luciferase reporter plasmids, two different regions (W1-1097: - 3697/ $2600 \mathrm{bp}$ and W2-736:

$-3336 /-2600 \mathrm{bp}$ ) that locate upstream of Prx-2 start codon were amplified by PCR. The amplified fragments were cloned into the pGL3-basic vector with KpnI and SacI (Promega). Candidate 1, W1-1097 (from -3697 to -2600), contains two NF- $\kappa$ B binding sites, while candidate 2, W2-736 (from -3336 to -2600$)$, contains one $\mathrm{NF}-\kappa \mathrm{B}$ binding site. Site-specific mutations of the NF- $\kappa \mathrm{B}$ binding sites in the promoter were designed on the basis of published data (9). For mutational analysis, three mutated constructs (M1-1097, M2-736, and M3-1097) were cloned into the pGL3-basic vector. In M1-1097 plasmid, one NF- $\kappa \mathrm{B}$ binding site that locates at -3361 / -3352 bp from Prx-2 start codon was mutated. In the M2-736 plasmid, the other NF- $\kappa \mathrm{B}$ binding site that locates at $-3254 /-3245$ bp from Prx-2 start codon was mutated. In the M3-1097 plasmid, both NF- $\kappa$ B binding sites were mutated by PCR-based site-directed mutagenesis. The following primers were used to construct the luciferase reporters containing various regions of Prx-2 promoter or mutated NF- $\kappa$ B binding sites (the underlined sequences indicate the mutations):

pGL3-Prx-2/W1-1097 forward, 5'-AGG TAC CGT CTC CTA CTA CCT CCA TTC-3';

pGL3-Prx-2/W1-1097 reverse, 5'-TTG AGC TCG CAA TGC TAG TCA GAA CTG-3';

pGL3-Prx-2/W2-736 forward, 5'-ATG GTA CCC CTG TTT AGG CTC TGC A-3';

pGL3-Prx-2/W2-736 reverse, 5'-TTG AGC TCG CAA TGC TAG TCA GAA CTG-3';

pGL3-Prx-2/M1-1097 forward, 5'-TAC CAC TGT CTC AGA TGC CGG-3';

pGL3-Prx-2/M1-1097 reverse, 5'-CCG GCA TCT GAG ACA GTG GTA-3';

pGL3-Prx-2/M2-736 forward, 5'-TTA GGA AGC TGA

GAT CTC CCG GA-3';

and pGL3-Prx-2/M2-736 reverse, 5'-TCC GGG AGA TCT CAG CTT CCT AA-3'.

\section{Luciferase assay}

Cells were transfected with $0.5 \mu \mathrm{g}$ of the pGL3-basic derived plasmids along with the internal control plasmid, pSV$\beta$-gal (Promega). Cells were incubated in PM or DM, and harvested in lysis buffer. Luciferase and $\beta$-gal activities were measured by a microplate reader (Bio-Rad, Richmond, CA), using $20 \mu \mathrm{L}$ of each cell lysate. The luciferase activity was normalized on the basis of $\beta$-galactosidase ( $\beta$-gal) activity.

\section{Preparation of nuclear extracts}

Nuclear extracts were obtained as previously described with minor modifications (28). Confluent cells were detached 
by trypsinization and centrifuged at $90 \mathrm{~g}$ at $4^{\circ} \mathrm{C}$ for $5 \mathrm{~min}$. Cells were resuspended in PBS, centrifuged at $20,000 \mathrm{~g}$ at $4^{\circ} \mathrm{C}$ for $5 \mathrm{~min}$, and once again resuspended in $200 \mu \mathrm{L}$ of buffer (10 mM Hepes, pH 7.9, $10 \mathrm{mM} \mathrm{KCl,} 1 \mathrm{mM}$ DTT, 0.5 mM PMSF, $0.1 \mathrm{mM}$ EDTA). After incubation on ice for $10 \mathrm{~min}$, cells were lysed by addition of $12.5 \mu \mathrm{L}$ of $10 \%$ NP-40. Harvesting of the nuclei was accomplished by centrifugation at $20,000 \mathrm{~g}$ at $4^{\circ} \mathrm{C}$ for $2 \mathrm{~min}$. The supernatant (cytosolic extract) was transferred to a new tube, and the nuclear pellets were resuspended in $50 \mu \mathrm{L}$ of extraction buffer (20 mM Hepes, pH 7.9, $0.4 \mathrm{M} \mathrm{NaCl}$, $1 \mathrm{mM}$ DTT, $1 \mathrm{mM}$ PMSF, $1 \mathrm{mM}$ EDTA, 1\% NP-40) and incubated on ice for $10 \mathrm{~min}$. Nuclear debris was removed by centrifugation at $20,000 \mathrm{~g}$ at $4^{\circ} \mathrm{C}$ for $10 \mathrm{~min}$, and the supernatant was used as nuclear protein extract.

\section{Electrophoretic Mobility Shift Assay (EMSA)}

EMSA was performed as previously described (27) using oligonucleotides. The following oligonucleotides for the NF$\kappa \mathrm{B}$ consensus-binding sequences were used for EMSA: candidate 1 (5'-ACTGTGGGAGATGCCGGGCC-3') and candidate 2 (5'-TTTAGGAAGCTCCCATCTCC-3'). Sense and antisense strands of oligonucleotides were annealed into double-stranded oligonucleotides and labeled with $\left[\gamma-{ }^{32} \mathrm{P}\right]$ ATP. For a competition study, a 100 -fold molar excess of unlabeled oligonucleotides was added to the reaction mixture prior to the addition of radiolabeled probe.

\section{Chromatin immunoprecipitation (ChIP)}

Conventional ChIP was conducted as previously described (14), except that cross-linked C2C12 chromatin was subjected to immunoprecipitation with antibodies against NF- $\kappa \mathrm{B}$ p65 and NF- $\kappa \mathrm{B}$ p50. The Prx -2 promoters containing the NF- $\kappa \mathrm{B}$ binding sites were amplified by following primers:

candidate 1 forward, 5'-GCTGAGGCCTTCTGACTCACA TTT-3';

candidate 1 reverse, 5'-TAA ACAGGAAGCAGCTGG GGC-3',

candidate 2 forward, 5'-AGCTGCTTCCTGTTTAGGCT CTG-3';

and candidate 2 reverse, 5'-CGGGAGATGGGAGCTTC CTAAAA-3'.

\section{Prx-2 overexpression}

The wild-type Prx-2 gene was inserted into the pCMVtag2B vector with XhoI and EcoRI (Stratagene, La Jolla, CA). $\mathrm{C} 2 \mathrm{C} 12$ cells were transiently transfected with pCMV-tag2BPrx-2 or an empty pCMV-tag2B vector using TurboFect ${ }^{\text {TM }}$ in vitro Transfection Reagent (Fermentas, Burlington, Ontario, Canada) according to the manufacturer's instructions.

\section{RNA interference}

Small interfering RNA (siRNA) against Prx-2 and NF- $\kappa$ B p65, and scrambled siRNA (control) were purchased from Dharmacon (Chicago, IL). Transient transfection of C2C12 cells with siRNAs targeting Prx-2 (50 nmol/L) and NF- $\kappa \mathrm{B}$ p 65 $(100 \mathrm{nmol} / \mathrm{L})$, or scrambled siRNA $(50 \mathrm{nmol} / \mathrm{L}$ or $100 \mathrm{nmol} /$ L) was carried out with the Lipofectamine 2,000 transfection reagent (Invitrogen, San Diego, CA). Transient transfection of primary myoblasts with siRNAs targeting Prx-2 (25 nmol/L) or scrambled siRNA $(25 \mathrm{nmol} / \mathrm{L})$ was carried out with the Lipofectamine RNAiMAX reagent (Invitrogen). When cells reached $30 \%-50 \%$ confluence, they were transfected with each siRNA, and further incubated for $24-48 \mathrm{~h}$. When cells reached $80 \%$ confluence, they were induced to differentiate in DM.

\section{Western blot analysis}

Cells were lysed with RIPA buffer ( $50 \mathrm{mM}$ Tris- $\mathrm{HCl}$, $\mathrm{pH}$ 7.4, $150 \mathrm{mM} \mathrm{NaCl}, 5 \mathrm{mM}$ EDTA, 1\% Na-deoxycholate, 1\% NP40), supplemented with protease inhibitors (200 mM PMSF, $200 \mathrm{mM} \mathrm{Na}_{2} \mathrm{VO}_{4}, 200 \mathrm{mM} \mathrm{NaF}$ ). The TA muscles were lysed with protein extraction solution (iNtRON Biotechnology, Chinju, Korea). The lysates were separated by sodium dodecyl sulfate polyacrylamide gel electrophoresis (SDS-PAGE) and transferred onto a nitrocellulose membrane. After blocking, the membranes were incubated with the indicated primary antibodies, followed by incubation with secondary antibody. Samples were detected with enhanced chemiluminescence reagents (Santa Cruz Biotechnology). Quantification of Western blots was performed by densitometry using Image J software. All Western blot analyses were performed three times to ensure that the results were repeatable, and the representative blots were shown. $\beta$-actin was used as a loading control.

\section{Statistical analysis}

Results were expressed as the means \pm standard error (SE). Error bars represent the means $\pm \mathrm{SE}$ of at least three independent experiments. The difference between two mean values was analyzed by Student's $t$ - test, and considered statistically significant when $p<0.05$.

\section{Acknowledgments}

This work was supported by the National Research Foundation of Korea (NRF) grant funded by the Korean government (MEST) (No. 2011-0030721).

\section{Author Disclosure Statement}

No competing financial interests exist.

\section{References}

1. Bast A, Wolf G, Oberbaumer I, and Walther R. Oxidative and nitrosative stress induces peroxiredoxins in pancreatic beta cells. Diabetologia 45: 867-876, 2002.

2. Chiribau CB, Cheng L, Cucoranu IC, Yu YS, Clempus RE, and Sorescu D. FOXO3A regulates peroxiredoxin III expression in human cardiac fibroblasts. J Biol Chem 283: 82118217, 2008.

3. Choi MH, Lee IK, Kim GW, Kim BU, Han YH, Yu DY, Park HS, Kim KY, Lee JS, Choi C, Bae YS, Lee BI, Rhee SG, and Kang SW. Regulation of PDGF signalling and vascular remodelling by peroxiredoxin II. Nature 435: 347353, 2005.

4. Chowdhury I, Mo Y, Gao L, Kazi A, Fisher AB, and Feinstein SI. Oxidant stress stimulates expression of the human peroxiredoxin 6 gene by a transcriptional mechanism involving an antioxidant response element. Free Radic Biol Med 46: 146-153, 2009.

5. Conejo R, Valverde AM, Benito M, and Lorenzo M. Insulin produces myogenesis in $\mathrm{C} 2 \mathrm{C} 12$ myoblasts by induction of 
NF-kappaB and downregulation of AP-1 activities. J Cell Physiol 186: 82-94, 2001.

6. de Alvaro C, Martinez N, Rojas JM, and Lorenzo M. Sprouty-2 overexpression in $\mathrm{C} 2 \mathrm{C} 12$ cells confers myogenic differentiation properties in the presence of FGF2. Mol Biol Cell 16: 4454-4461, 2005.

7. Ding Y, Choi KJ, Kim JH, Han X, Piao Y, Jeong JH, Choe W, Kang I, Ha J, Forman HJ, Lee J, Yoon KS, and Kim SS. Endogenous hydrogen peroxide regulates glutathione redox via nuclear factor erythroid 2-related factor 2 downstream of phosphatidylinositol 3-kinase during muscle differentiation. Am J Pathol 172: 1529-1541, 2008.

8. Hayden MS and Ghosh S. Signaling to NF-kappaB. Genes Dev 18: 2195-2224, 2004.

9. Hoeflich KP, Luo J, Rubie EA, Tsao MS, Jin O, and Woodgett JR. Requirement for glycogen synthase kinase-3beta in cell survival and NF-kappaB activation. Nature 406: 86-90, 2000.

10. Immenschuh $S$ and Baumgart-Vogt E. Peroxiredoxins, oxidative stress, and cell proliferation. Antioxid Redox Signal 7: 768-777, 2005.

11. Jiang BH, Aoki M, Zheng JZ, Li J, and Vogt PK. Myogenic signaling of phosphatidylinositol 3-kinase requires the serine-threonine kinase Akt/protein kinase B. Proc Natl Acad Sci USA 96: 2077-2081, 1999.

12. Kabe Y, Ando K, Hirao S, Yoshida M, and Handa H. Redox regulation of NF-kappaB activation: Distinct redox regulation between the cytoplasm and the nucleus. Antioxid Redox Signal 7: 395-403, 2005.

13. Karihtala P, Mantyniemi A, Kang SW, Kinnula VL, and Soini Y. Peroxiredoxins in breast carcinoma. Clin Cancer Res 9: 3418-3424, 2003.

14. Kim J, Choi TG, Ding Y, Kim Y, Ha KS, Lee KH, Kang I, Ha J, Kaufman RJ, Lee J, Choe W, and Kim SS. Overexpressed cyclophilin B suppresses apoptosis associated with ROS and $\mathrm{Ca} 2+$ homeostasis after ER stress. J Cell Sci 121: 36363648, 2008.

15. Kim SY, Jo HY, Kim MH, Cha YY, Choi SW, Shim JH, Kim $\mathrm{TJ}$, and Lee KY. H2O2-dependent hyperoxidation of peroxiredoxin $6(\operatorname{Prd} \times 6)$ plays a role in cellular toxicity via up-regulation of iPLA2 activity. J Biol Chem 283: 3356333568, 2008.

16. Kim YJ, Ahn JY, Liang P, Ip C, Zhang Y, and Park YM. Human prx1 gene is a target of Nrf2 and is up-regulated by hypoxia/reoxygenation: implication to tumor biology. Cancer Res 67: 546-554, 2007.

17. Lee J, Choi KJ, Lim MJ, Hong F, Choi TG, Tak E, Lee S, Kim YJ, Chang SG, Cho JM, Ha J, and Kim SS. Proto-oncogenic $\mathrm{H}-\mathrm{Ras}, \mathrm{K}-\mathrm{Ras}$, and N-Ras are involved in muscle differentiation via phosphatidylinositol 3-kinase. Cell Res 20: 919-934, 2010.

18. Lee S, Tak E, Lee J, Rashid MA, Murphy MP, Ha J, and Kim SS. Mitochondrial $\mathrm{H} 2 \mathrm{O} 2$ generated from electron transport chain complex I stimulates muscle differentiation. Cell Res 21: 817-834, 2011.

19. Li J, Stouffs M, Serrander L, Banfi B, Bettiol E, Charnay Y, Steger K, Krause KH, and Jaconi ME. The NADPH oxidase NOX4 drives cardiac differentiation: Role in regulating cardiac transcription factors and MAP kinase activation. Mol Biol Cell 17: 3978-3988, 2006.

20. Lim MJ, Choi KJ, Ding Y, Kim JH, Kim BS, Kim YH, Lee J, Choe W, Kang I, Ha J, Yoon KS, and Kim SS. RhoA/Rho kinase blocks muscle differentiation via serine phosphorylation of insulin receptor substrate-1 and -2. Mol Endocrinol 21: 2282-2293, 2007.
21. Livak KJ and Schmittgen TD. Analysis of relative gene expression data using real-time quantitative PCR and the 2(-Delta Delta C(T)) method. Methods 25: 402-408, 2001.

22. Merkhofer EC, Cogswell P, and Baldwin AS. Her2 activates NF-kappaB and induces invasion through the canonical pathway involving IKKalpha. Oncogene 29: 1238-1248, 2010.

23. Miyamoto N, Izumi H, Miyamoto R, Kondo H, Tawara A, Sasaguri $\mathrm{Y}$, and Kohno K. Quercetin induces the expression of peroxiredoxins 3 and 5 via the Nrf2/NRF1 transcription pathway. Invest Ophthalmol Vis Sci 52: 1055-1063, 2011.

24. Mowbray AL, Kang DH, Rhee SG, Kang SW, and Jo H. Laminar shear stress up-regulates peroxiredoxins (PRX) in endothelial cells: PRX 1 as a mechanosensitive antioxidant. J Biol Chem 283: 1622-1627, 2008.

25. Nakano H, Nakajima A, Sakon-Komazawa S, Piao JH, Xue X, and Okumura K. Reactive oxygen species mediate crosstalk between NF-kappaB and JNK. Cell Death Differ 13: 730-737, 2006.

26. Olahova M, Taylor SR, Khazaipoul S, Wang J, Morgan BA, Matsumoto K, Blackwell TK, and Veal EA. A redox-sensitive peroxiredoxin that is important for longevity has tissue- and stress-specific roles in stress resistance. Proc Natl Acad Sci USA 105: 19839-19844, 2008.

27. Pantano C, Reynaert NL, van der Vliet A, and JanssenHeininger YM. Redox-sensitive kinases of the nuclear factorkappaB signaling pathway. Antioxid Redox Signal 8: 1791-1806, 2006.

28. Piao YJ, Seo YH, Hong F, Kim JH, Kim YJ, Kang MH, Kim BS, Jo SA, Jo I, Jue DM, Kang I, Ha J, and Kim SS. Nox 2 stimulates muscle differentiation via NF-kappaB/iNOS pathway. Free Radic Biol Med 38: 989-1001, 2005.

29. Rhee SG, Chang TS, Bae YS, Lee SR, and Kang SW. Cellular regulation by hydrogen peroxide. J Am Soc Nephrol 14: S211215, 2003.

30. Rhee SG, Yang KS, Kang SW, Woo HA, and Chang TS. Controlled elimination of intracellular $\mathrm{H}(2) \mathrm{O}(2)$ : Regulation of peroxiredoxin, catalase, and glutathione peroxidase via post-translational modification. Antioxid Redox Signal 7: 619-626, 2005.

31. Shau H, Butterfield LH, Chiu R, and Kim A. Cloning and sequence analysis of candidate human natural killer-enhancing factor genes. Immunogenetics 40: 129-134, 1994.

32. Storz P, Doppler H, and Toker A. Protein kinase D mediates mitochondrion-to-nucleus signaling and detoxification from mitochondrial reactive oxygen species. Mol Cell Biol 25: 8520-8530, 2005.

33. Sumitani S, Goya K, Testa JR, Kouhara H, and Kasayama S. Akt1 and Akt2 differently regulate muscle creatine kinase and myogenin gene transcription in insulin-induced differentiation of C2C12 myoblasts. Endocrinology 143: 820828, 2002.

34. Veal EA, Day AM, and Morgan BA. Hydrogen peroxide sensing and signaling. Mol Cell 26: 1-14, 2007.

35. Yang HY, Kwon J, Cho EJ, Choi HI, Park C, Park HR, Park $\mathrm{SH}$, Chung KJ, Ryoo ZY, Cho KO, and Lee TH. Proteomic analysis of protein expression affected by peroxiredoxin $\mathrm{V}$ knock-down in hypoxic kidney. J Proteome Res 9: 4003-4015, 2010.

36. Yun SJ, Seo JJ, Chae JY, and Lee SC. Peroxiredoxin I and II are up-regulated during differentiation of epidermal keratinocytes. Arch Dermatol Res 296: 555-559, 2005. 


\author{
Address correspondence to: \\ Dr. Sung Soo Kim \\ Department of Biochemistry and Molecular Biology \\ School of Medicine \\ Kyung Hee University \\ \#1, Hoegi-dong \\ Dongdaemoon-gu \\ Seoul 130-701 \\ Republic of Korea \\ E-mail: sgskim@khu.ac.kr
}

Date of first submission to ARS Central, February 17, 2011; date of final revised submission, August 30, 2011; date of acceptance, September 1, 2011.

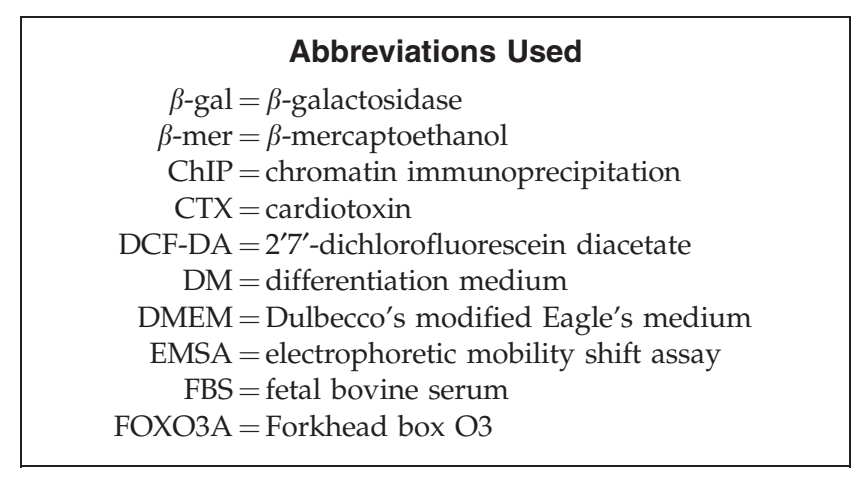

$\mathrm{H}$ and $\mathrm{E}=$ hematoxylin and eosin

HEK = human embryonic kidney

IGF $=$ insulin-like growth factor

$\mathrm{MEF}=$ mouse embryonic fibroblasts

$\mathrm{MHC}=$ myosin heavy chain

$\mathrm{NAC}=\mathrm{N}$-acetyl L-cysteine

$\mathrm{NEM}=\mathrm{N}$-ethylmaleimide

$\mathrm{NF}-\kappa \mathrm{B}=$ nuclear factor-kappa $\mathrm{B}$

Nrf2 $=$ erythroid 2-related factor 2

PDTC $=$ pyrrolidinedithiocarbamate acid

$\mathrm{PI} 3 \mathrm{~K}=$ phosphatidylinositol 3-kinase

PLC $-\gamma 1=$ Phospholipase C $-\gamma 1$

$\mathrm{PM}=$ proliferation medium

Prx-2 $=$ peroxiredoxin 2

Prxs $=$ peroxiredoxins

ROS $=$ reactive oxygen species

RT-PCR $=$ reverse transcription-PCR

SDS-PAGE $=$ sodium dodecyl sulfate polyacrylamide gel electrophoresis

$\mathrm{SE}=$ standard error

siRNA $=$ small interfering RNA

Srx $=$ sulfiredoxin

$\mathrm{TA}=$ tibialis anterior

Tiron $=4,5$-dihydroxy-1,3-benzenedisulfonic acid

$\operatorname{Trx}=$ thioredoxin

$\operatorname{Trx}-1=$ thioredoxin 1 
